\title{
OPEN Phylogenetic and biochemical analysis of calsequestrin structure and association of its variants with cardiac disorders
}

\begin{abstract}
Qian Wang ${ }^{1}$, Tautvydas Paskevicius ${ }^{1}$, Alexander Filbert ${ }^{2}$, Wenying Oin ${ }^{3}$, Hyeong Jin $\mathrm{Kim}^{1}$, Xing-Zhen Chen ${ }^{3,4}$, Jingfeng Tang ${ }^{3}$, Joel B. Dacks ${ }^{2 \bowtie}$, Luis B. Agellon ${ }^{5 \bowtie}$ \& Marek Michalak $^{1,3 \bowtie}$

Calsequestrin is among the most abundant proteins in muscle sarcoplasmic reticulum and displays a high capacity but a low affinity for $\mathrm{Ca}^{2+}$ binding. In mammals, calsequestrin is encoded by two genes, CASO1 and CASO2, which are expressed almost exclusively in skeletal and cardiac muscles, respectively. Phylogenetic analysis indicates that calsequestrin is an ancient gene in metazoans, and that the duplication of the ancestral calsequestrin gene took place after the emergence of the lancelet. CASQ2 gene variants associated with catecholaminergic polymorphic ventricular tachycardia (CPVT) in humans are positively correlated with a high degree of evolutionary conservation across all calsequestrin homologues. The mutations are distributed in diverse locations of the calsequestrin protein and impart functional diversity but remarkably manifest in a similar phenotype in humans.
\end{abstract}

The sarcoplasmic reticulum (SR) is a high specialized membrane network that supports mechanical muscle functions requiring large fluxes of $\mathrm{Ca}^{2+}$. Consequently, the SR controls excitation-contraction coupling ${ }^{1-3}$ without compromising $\mathrm{Ca}^{2+}$ requiring cellular processes that are normally associated with the endoplasmic reticulum ${ }^{4}$. There are two well defined structural and functional regions of the SR in cardiac muscle: the longitudinal SR that runs parallel to the myofibrils and the junctional SR that forms multiple membrane contacts with T-tubule membrane contact sites ${ }^{5-7}$. SR-T-tubules membrane contact sites play an important role in initiation of the excitation-contraction coupling ${ }^{7}$. The longitudinal SR is enriched with $\mathrm{Ca}^{2+}$-ATPase (SERCA) which is responsible for the rapid removal of $\mathrm{Ca}^{2+}$ from the cytoplasm to initiate muscle relaxation ${ }^{1,5,6}$. The junctional SR is the primary site of $\mathrm{Ca}^{2+}$ release via the Ryanodine Receptor (RyR)/Ca ${ }^{2+}$ channel which triggers muscle contraction. The SR luminal $\mathrm{Ca}^{2+}$ binding protein calsequestrin is a high capacity, low affinity $\mathrm{Ca}^{2+}$ binding protein that forms oligomeric structures that regulate RyR activity via interactions with RyR, triadin and junctin ${ }^{1,8-14}$. There are two isoforms of calsequestrin, which are encoded by two different genes: cardiac calsequestrin (CASQ2) and skeletal muscle calsequestrin $(C A S Q 1)^{14,15}$. The crystal structure of calsequestrin indicates that the protein contains three thioredoxin-like domains reminiscent of ER luminal oxidoreductases ${ }^{16,17}$.

Catecholaminergic polymorphic ventricular tachycardia (CPVT) is an inherited disease characterized by ventricular arrhythmias leading to sudden death ${ }^{18,19}$. CPVT results from defects in intracellular $\mathrm{Ca}^{2+}$ handling by cardiomyocytes. Two major variants have been associated with the CPVT disorder. The autosomal dominant form is associated with mutations in the cardiac ryanodine receptor (RyR2) gene and accounts for $~ 50 \%$ cases, while a recessive form with mutations in the cardiac isoform of calsequestrin (CASQ2) accounts for $2-5 \%$ cases. Other mutations also found in the CALM1, CALM2, CALM3 (encodes calmodulin1, 2, 3 respectively), and TRDN (encodes triadin) gene account for $<2 \%$ of CPVT $\operatorname{cases}^{20-24}$. Thirteen mutations in the CASQ2 gene have been identified in CPVT patients, in sudden death syndrome ${ }^{25,26}$, and three of them are non-synonymous polymorphisms (cSNP) ${ }^{24,27}$. Several biochemical and cell biological studies of R33Q, L167H, and D307H calsequestrin

\footnotetext{
${ }^{1}$ Department of Biochemistry, University of Alberta, Edmonton, Alberta T6G 2H7, Canada. ${ }^{2}$ Division of Infectious Disease, Department of Medicine, University of Alberta, Edmonton, AB T6G 2G3, Canada. ${ }^{3}$ Institute of Biomedical and Pharmaceutical Sciences, Key Laboratory of Fermentation Engineering, Hubei Provincial Cooperative Innovation Center of Industrial Fermentation, Hubei Key Laboratory of Industrial Microbiology, Hubei University of Technology, Wuhan, Hubei, China. ${ }^{4}$ Department of Physiology, University of Alberta, Edmonton, Alberta T6G 2H7, Canada. ${ }^{5}$ School of Dietetics and Human Nutrition, McGill University, Ste. Anne de Bellevue, Quebec H9X 3V9, Canada.『email: dacks@ualberta.ca; luis.agellon@mcgill.ca; marek.michalak@ualberta.ca
} 
mutants indicate that these mutations lead to impaired $\mathrm{Ca}^{2+}$ storage and $\mathrm{Ca}^{2+}$ release from the $\mathrm{SR}^{22,24-41}$. Recently new calsequestrin mutations have been identified including K180R, D351G, G332R, and P329S 27,39,40,42-46.

In this study, we examined the evolutionary constraints of the CPVT related calsequestrin mutations, and included Casq1, Casq2 and pre-duplicate calsequestrin in the phylogenetic analysis. We showed that calsequestrin is an ancient protein in the metazoan, and that the duplication of the calsequestrin gene took place after the divergence of the lancelet but before divergence of Chondrichthyes. We noted that calsequestrin mutations, associated with CPVT, positively correlated with an increase in the degree of evolutionary conservation of the mutated sites. Furthermore, we carried out biochemical and biophysical analysis of seven CPVT related mutants (R33Q, L167H, D307H, K180R), and whole exome sequencing variants (D351G, G332R, P329S) selected based on their linked to the human CPVT phenotype and located within highly conserved thioredoxin-like domain. The mutations are distributed in diverse locations of the calsequestrin protein but remarkably manifest in a similar phenotype in humans.

\section{Results}

Emergence and specialization of calsequestrin within animals. Phylogenetic analysis of the two calsequestrin genes (casq1 and casq2) was carried out with the aim of clarifying the distribution and conservation of each paralogue across animals, and thus deduce the timing of the gene duplication event and relate this information to calsequestrin mutants responsible for CPVT. Homology searching was undertaken in 23 metazoan genomes and three outgroup lineages to identify calsequestrin homologues. We identified unambiguous calsequestrin homologues in most of the vertebrate and invertebrate lineages (Supp. Table S1). Furthermore, we revealed that Ciona intestinalis, $B$. floridae, and all taxa within the invertebrates possess a single calsequestrin gene, including taxa as deeply branched as Trichoplax and Nematostella (Fig. 1). We did not identify a calsequestrin homologue in the sponge Amphimedon despite rigorous searches. This is potentially due to a database error, but more intriguingly may well represent a loss of calsequestrin in this lineage, since preliminary searches of other sponge genomes also failed to identify calsequestrin homologues. Although the relative branching order of the basal lineages within animals is still disputed, with Nematostella or sponges as the deepest branch, calsequestrin is clearly an ancient protein within the metazoan (Suppl. Figs. S1 and S2).

Each of these lineages, as well the invertebrates and hemichordates, possess only a single calsequestrin gene, leaving the timing of when casq1 vs casq 2 arose as an outstanding question. Preliminary phylogeny provided moderate support for the non-vertebrate sequences emerging basal to clades of the casq 1 and casq2, thus being pre-duplicated versions (Suppl. Figs. S1). Further analysis focused on vertebrate gene sequences and using the lancelet sequences as an outgroup (Suppl. Fig. S2). The analysis robustly showed that the calsequestrin gene duplication that gave rise to casq1 and casq2 occurred after the divergence of the lancelet lineage but before the divergence of the Chondrichthyes (i.e. cartilaginous fish). We were unable to identify any calsequestrin sequences in the insect lineage. This likely represents a bona fide loss in this line given the positive identification of a homologue in the spider Parasteatoda tepidariorum.

We believe the Chondrichthyes and Avian taxa lost casq1 independently. We identified casq1 paralogues in the Chondrichthyes despite robustly classifying casq2 being present and the duplication having taken place prior to this point (Suppl. Fig. S2). The same was observed for the avian taxa sampled, suggesting that casq1 was lost independently in these three lineages. In mammalian muscles the two calsequestrin isoforms exhibit tissue specific expression ${ }^{47-49}$. Casq2 is expressed in cardiac and slow-twitch skeletal muscle, whereas Casq1 is expressed in adult fast-twitched muscle ${ }^{47-49}$. Cartilaginous fish as well as avian animals have both fast-twitch and slow-twitch skeletal muscles even though they appear to lack casq1.

Conservation of CPVT associated Casq2 mutants throughout animal kingdom. Having the evolutionary distribution of casq1 and casq2 allowed us to assess the conservation of sites in Casq2 proteins where calsequestrin mutations in the human CASQ2, have been associated with CPVT (Fig. 2A,B $)^{24,50,51}$. We selected the following seven Casq2 mutants for further analyses: R33Q, L167H, D307H, K180R, P329S, G332R, and D351G (Fig. 2A,B). Many mutations are scattered across the three thioredoxin-like domains of Casq2 (Fig. 2A), but remarkably they all lead to a similar clinical outcome ${ }^{24,50}$. L167H, and D351G are conserved in Casq1 and Casq2 paralogues found in vertebrates but are variable in the pre-duplicated non-bilaterian (Fig. 2A). In contrast, positions R33Q, K180R, D307H, P329S and G332R are fully conserved across all calsequestrin homologues including pre-duplication Casq (Suppl. Fig. S2).

$\mathrm{Ca}^{2+}$ binding to Casq2 mutants. With the conservation of the relevant amino acid positions in hand, we next carried out biochemical and biophysical analysis of the Casq2 mutants in order to gain insight into the contribution of these mutations to the development of CPVT. First, we used microscale thermophoresis (MST) to investigate $\mathrm{Ca}^{2+}$ binding to Casq2 mutants. Mutation of Casq2 residues R33Q, L167H, K180R and D351G had no significant effect on $\mathrm{Ca}^{2+}$ binding to calsequestrin (Fig. $3 \mathrm{~A}-\mathrm{D}$ ) with $\mathrm{K}_{\mathrm{d}}$ values ranging from $0.872 \pm 0.283 \mathrm{mM}$ for wild-type to $1.052 \pm 0.154 \mathrm{mM}$ for the D351G mutant (Fig. 3H). However, P329S and G332R mutants exhibited altered $\mathrm{Ca}^{2+}$ binding affinities (Fig. 3E,F). Previous studies showed that R33Q, L167H, and D307H have highly reduced $\mathrm{Ca}^{2+}$ binding capacity ${ }^{17,31}$. Here, using label-free MST assay the D307H Casq2 mutant showed no measurable $\mathrm{Ca}^{2+}$ binding (Fig. 3G). Of the seven mutants associated with CPVT examined, only three exhibited altered $\mathrm{Ca}^{2+}$ binding properties.

Conformational changes and protein folding of Casq2 mutants. Casq2 undergoes conformational change upon $\mathrm{Ca}^{2+}$ binding, and this was monitored by circular dichroism (CD) analysis ${ }^{52}$. Upon adding $\mathrm{Ca}^{2+}$, wild-type Casq2 lost 18.3\% $\alpha$-helix and gained 16.79\% $\beta$-sheet conformation (Fig. 4) ${ }^{52}$. CD spectra for K180R 


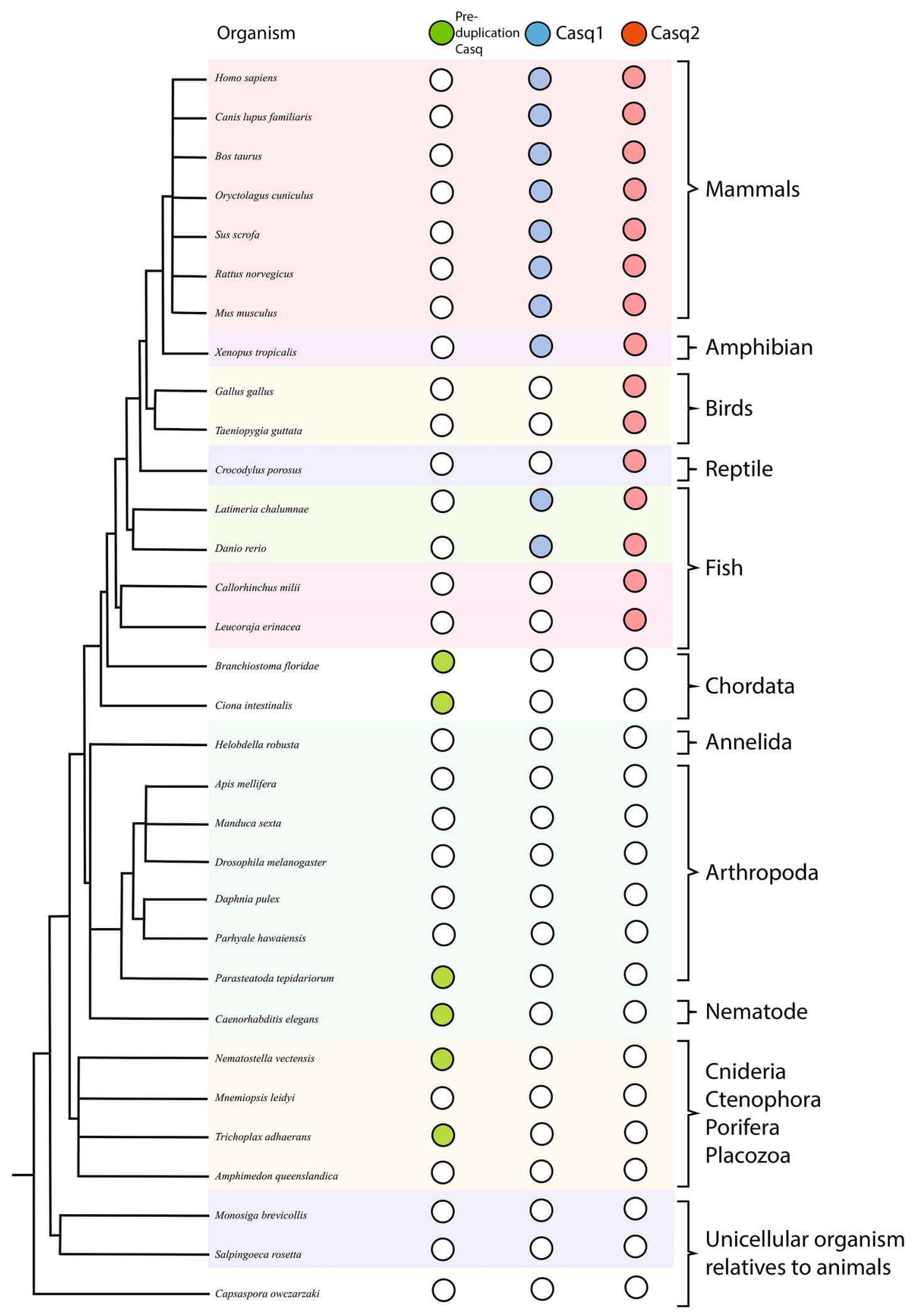

Figure 1. Calsequestrin homologues in the vertebrate and invertebrate lineages. Empty circles indicate the gene is absent. Green circles indicate the presence of pre-duplication Casq, blue circles the presence of casq1, and red circles the presence of casq2. 
A
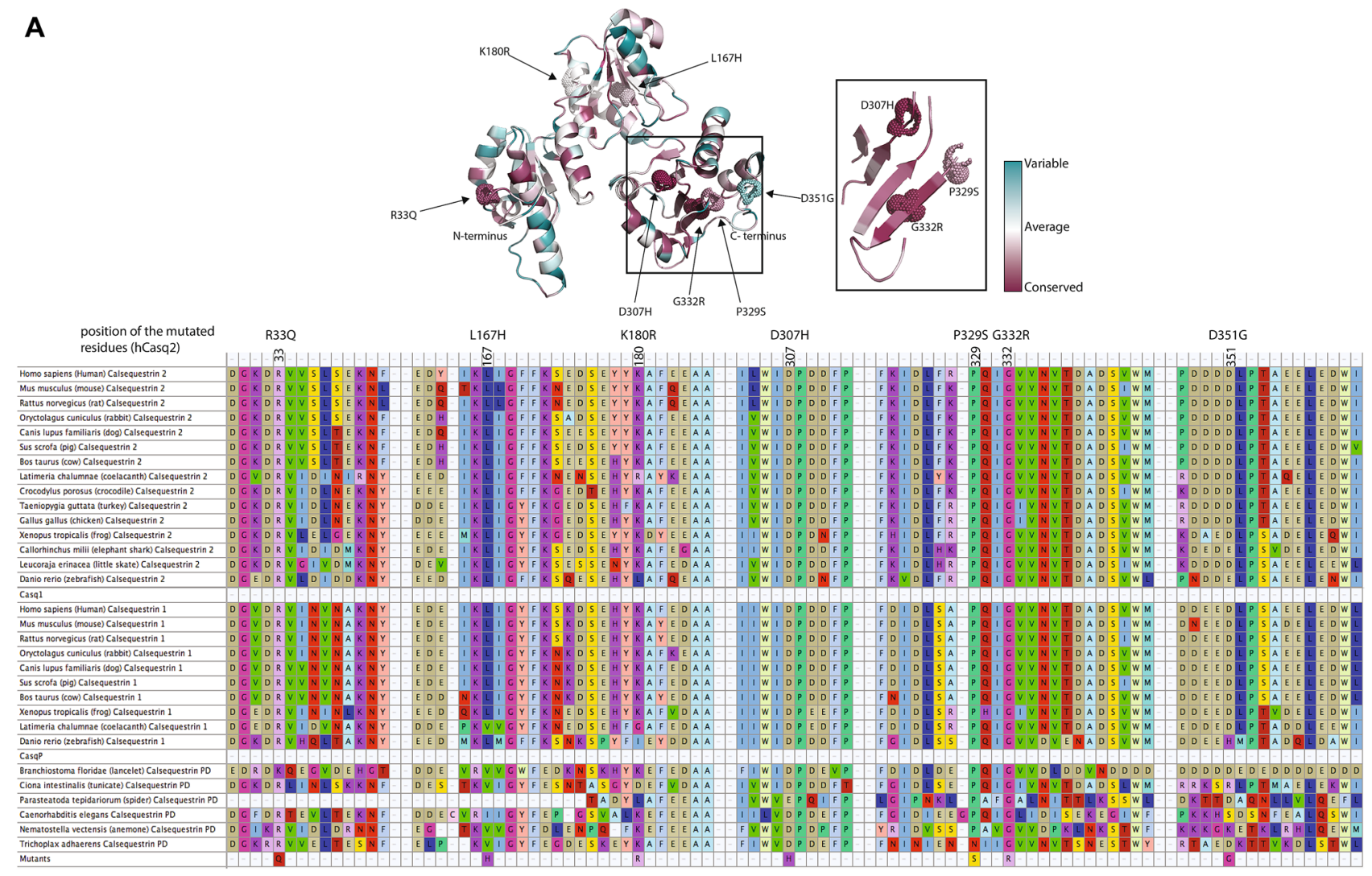

Figure 2. Amino acid sequence alignments and calsequestrin $3 \mathrm{D}$ structure. (A) The 3D structure of the cardiac isoform of calsequestrin (Casq2) is shown (2VAF). The location of R33Q, L167H, K180R, D307H, P329S, G332R, and D351G Casq2 mutants are depicted in the structure. A scale of variable to conserved residues is indicated in the Figure. Mutants are shown as dot sphere. The highly conserved beta-sheet from the third thioredoxin-like fold is enlarged in the box and shown separately. The table shows the alignment of calsequestrin amino acid sequences. The degree of conservation of Casq 2 sequences is color-coded using ConSurf ${ }^{71}$. Multiple sequence alignments were inputed from the Casq1, Casq2, and Casqp alignment. Different colors represent similar/identical amino acid residues. The location of mutated residues is indicated in the table. hCASQ2, human Casq2. (B) Human Casq2 (PBD: 2VAF) with electrostatic potential surfaces. The location of the N-and $\mathrm{C}$-terminus of Casq2 is indicated. Secondary structures are shown as green ribbons, the amino acid residues corresponding to the CPVT related mutations are indicated by the corresponding van der Waals surface as orange spheres. Local environments around R33, L167, K180, D307, P329, G332, and D351 residues are shown. Secondary structures are shown as green ribbons, dotted lines indicate a polar interaction between side-chains. Oxygen, nitrogen, and carbon atoms are shown in red, blue, and white, respectively. For G332, the hydrogens on a-carbon are shown in white. Prepared using PyMOL v2.4.1 (https://www.pymol.org/2/).

and D351G mutants overlapped with those of the wild-type Casq2 (Fig. 4C,D,E,F), indicating no effect of the K180R and D351G mutation on the protein conformation. However, the G332R and P329S mutants, showed altered sensitivity to $\mathrm{Ca}^{2+}$-induced conformational changes (Fig. 4E,F). This was in agreement with the reduced $\mathrm{Ca}^{2+}$ affinity of these mutants (Fig. 3). In contrast, the CD spectra of mutants R33Q, L167H, and D307H revealed increased $\alpha$-helix content that was not sensitive to addition of $\mathrm{Ca}^{2+}$ (Fig. 4A,B,G; Table 1).

Next, we tested susceptibility of the Casq2 and Casq2 mutants to trypsin digestion to further analyze the impact of Casq2 mutations on protein folding. K180R and D351G mutants showed trypsin digestion patterns similar to wild-type protein indicating no major folding differences between these proteins (Fig. 5). In support of the CD analysis, R33Q, L167H, and D307H mutants showed limited trypsin susceptibility compared to wild-type protein both in the absence and presence of $\mathrm{Ca}^{2+}$ (Fig. 5). R33Q, L167H, and D307H mutants showed more $\alpha$-helix (Fig. 4) and an increased sensitivity to trypsin digestion in the absent of $\mathrm{Ca}^{2+}$ (Fig. 5), indicative of altered protein folding. In agreement with $\mathrm{Ca}^{2+}$ binding (Fig. 3E,F) and $\mathrm{CD}$ analysis (Fig. 4E,F) trypsin digestion of P329S and G332R mutants also showed increased kinetics of digestion in the presence of Ca ${ }^{2+}$ (Fig. 5C,D), indicative of $\mathrm{Ca}^{2+}$-induced conformational changes of these mutants.

In agreement with previous study ${ }^{31}$, we found $\mathrm{D} 307 \mathrm{H}$ to be more susceptible to trypsin digestion at high $\mathrm{Ca}^{2+}$ concentration, and conformational changes of this mutant were less sensitive to increased $\mathrm{Ca}^{2+}$ concentration (Figs. 4 and 5). In absence of $\mathrm{Ca}^{2+}, \mathrm{D} 307 \mathrm{H}$ was shown to have trypsin susceptibility and CD spectra similar to wild-type protein ${ }^{17,31}$. In the present study we found $\mathrm{D} 307 \mathrm{H}$ protein was more sensitive to trypsin digestion (Fig. 5). This was further supported with altered CD spectra of the D307H mutant (Fig. 4; Table 1). The 


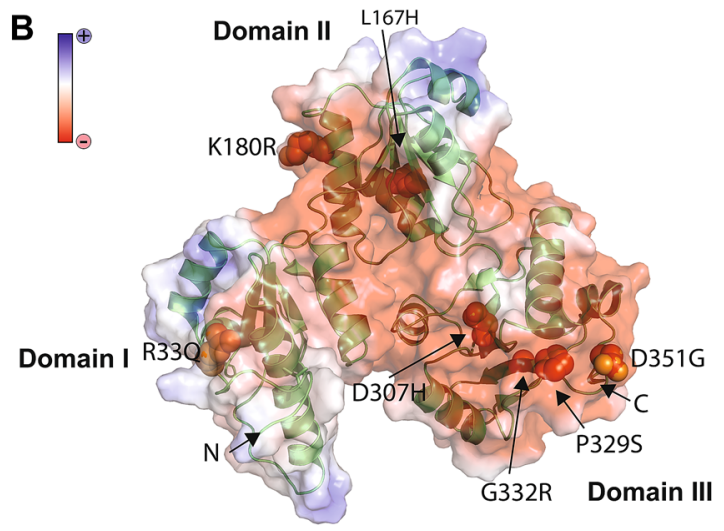

K180

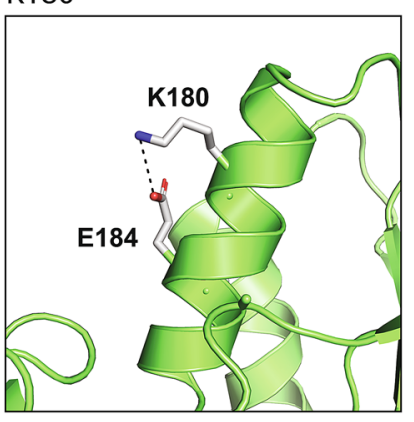

D307

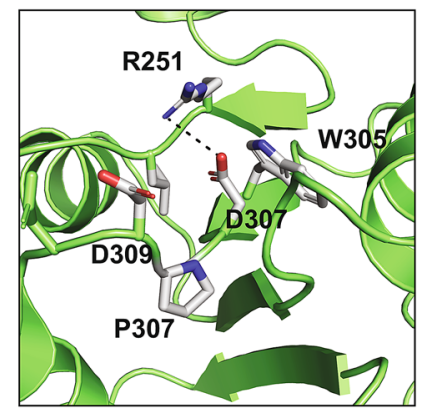

R33

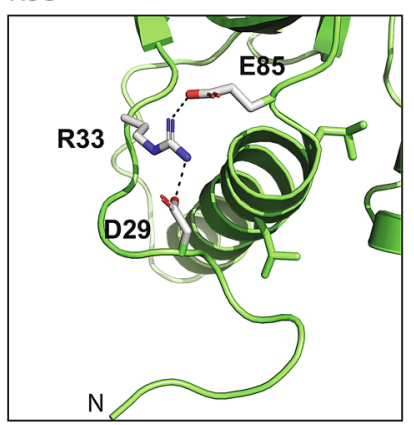

P329 and D351

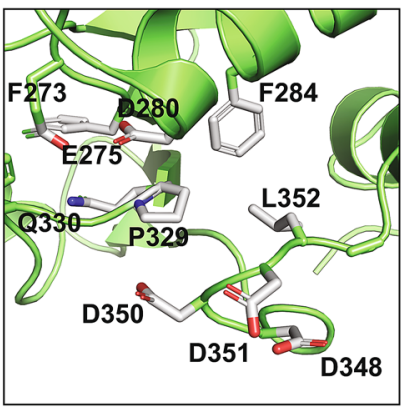

L167

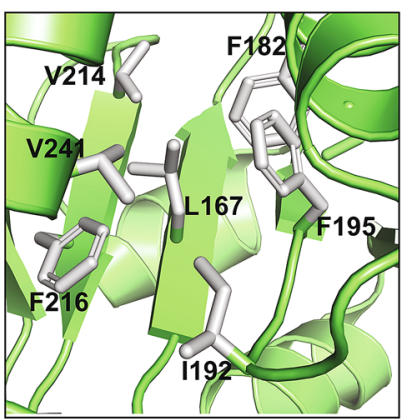

Figure 2. (continued)

differences between the two studies is likely due to different buffer conditions used for CD analysis and trypsin digestion. As previously reported ${ }^{53}, \mathrm{~L} 167 \mathrm{H}$ and $\mathrm{R} 33 \mathrm{Q}$ were more susceptible to trypsin digestion in absence $\mathrm{Ca}^{2+}$ or presence of $\mathrm{Ca}^{2+}$ indicating altered protein folding of the mutants. This was supported by CD analysis (Fig. 4) and thermal denaturation assays (Fig. 6; Table 2).

Next, we used the Tycho NT.6 system to carry out thermal denaturation analysis of wild-type Casq2 and Casq2 mutants in a label-free environment as another indicator of protein folding. The analysis is based on measurement of the protein's intrinsic tryptophan fluorescence and records a protein's unfolding profile in real-time. Casq2 has 5 tryptophan residues all located in the third thioredoxin-like domain ${ }^{16,17}$ and fully buried in the hydrophobic core. K180R and D351G mutants showed an unfolding profile (Fig. 6A) and inflection temperature (Ti, proportionally to protein melting temperature) values (Fig. 6F,G; Table 2) similar to wild-type Casq2. Ti values for R33Q $\left(52.55^{\circ} \mathrm{C}\right)$ and $\mathrm{G} 332 \mathrm{R}\left(47.23^{\circ} \mathrm{C}\right)$ mutants, although close to the wild-type Casq2 $\left(49.89^{\circ} \mathrm{C}\right)$, were statistically different (Fig. 6C,E). P329S, G332R and D307H mutants showed minimal (for P329S and G332R mutants) to no detectable (for D307H mutant) unfolding transition (Fig. 6; Table 2). These mutants also showed a significantly higher initial ratio $\left(350 \mathrm{~nm} / 330 \mathrm{~nm}\right.$ at $\left.35^{\circ} \mathrm{C}\right)$, indicating that tryptophan residues in P329S, G332R and D307H mutants were exposed to solvent, and the polarity of the local tryptophan environment was unchanged upon denaturing at higher temperature. The $\mathrm{L} 167 \mathrm{H}$ mutant had an intermediate unfolding profile and significantly increased Ti value (Fig. 6; Table 2), indicating partially exposed tryptophan. The L167H mutation resulted in a partial disruption of the third thioredoxin-like domain and/or destabilized another protein domain, whereas, D307H, P329S, and G332R exhibited a large disruption in the third thioredoxin-like domain (Fig. 6; Table 2).

$\mathrm{Ca}^{2+}$ dependent polymerization of Casq2 mutants. Casq2 undergoes monomer to oligomer transition and oligomerization ${ }^{54}$. Upon binding to $\mathrm{Ca}^{2+}$, Casq2 undergoes reversible polymerization, and this affects Casq2 assembly to the junctional SR, which could have direct impact on SR $\mathrm{Ca}^{2+}$ supply and RyR2 regulation ${ }^{54}$. We tested for a $\mathrm{Ca}^{2+}$-dependent oligomerization of Casq2 mutants using disuccinimidyl suberate (DSS) crosslinker (Fig. 7) and native gel electrophoresis techniques (Fig. 8). Addition of $\mathrm{Ca}^{2+}$ to wild-type Casq2 increased oligomerization of the protein (Fig. 7). A similar pattern of $\mathrm{Ca}^{2+}$-dependent oligomerization was seen for K180R, D351G and D307H mutants (Fig. 7). Surprisingly, the D307H mutant that did not bind $\mathrm{Ca}^{2+}$ (Fig. 3) but showed $\mathrm{Ca}^{2+}$-dependent oligomerization indistinguishable from the wild-type Casq2 (Fig. 7) suggesting a role of $\mathrm{Ca}^{2+}$ in function of this mutant. R33Q, L167H, P329S and G332R mutants had increased $\mathrm{Ca}^{2+}$-dependent oligomerization whereas R33Q mutant showed no dependence on $\mathrm{Ca}^{2+}$ for oligomerization (Fig. 7). Under conditions of native electrophoresis, wild-type Casq2 and Casq2 mutants exhibited spontaneous oligomerization (Fig. 8) with R33Q, L167H and G332R mutants having a greater proportion in oligomeric form as compared to wild-type protein (Fig. 8). This was particularly evident for the R33Q and L167H mutants which existed predominantly ( $>80 \%$ and $>60 \%$, respectively) in an oligomeric form (Fig. 8 ). 

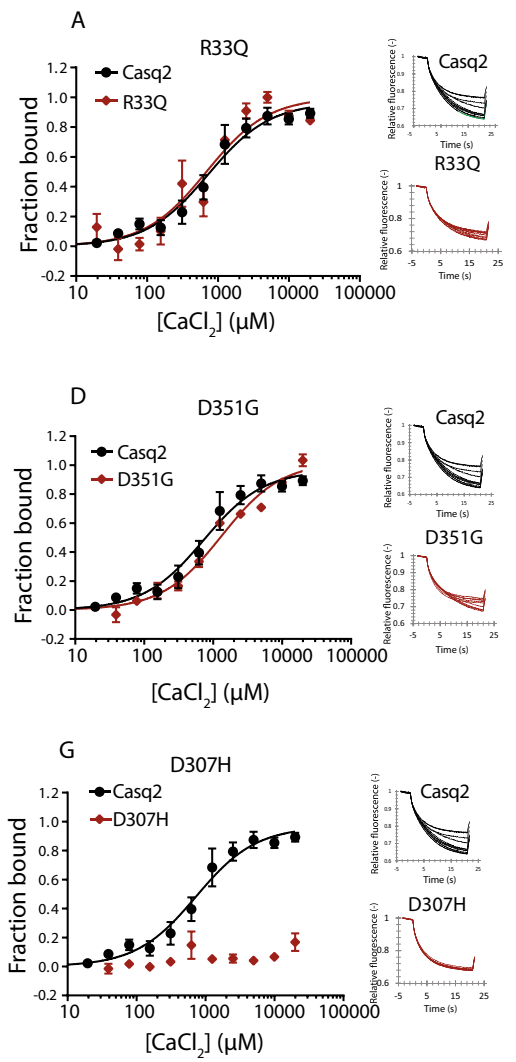
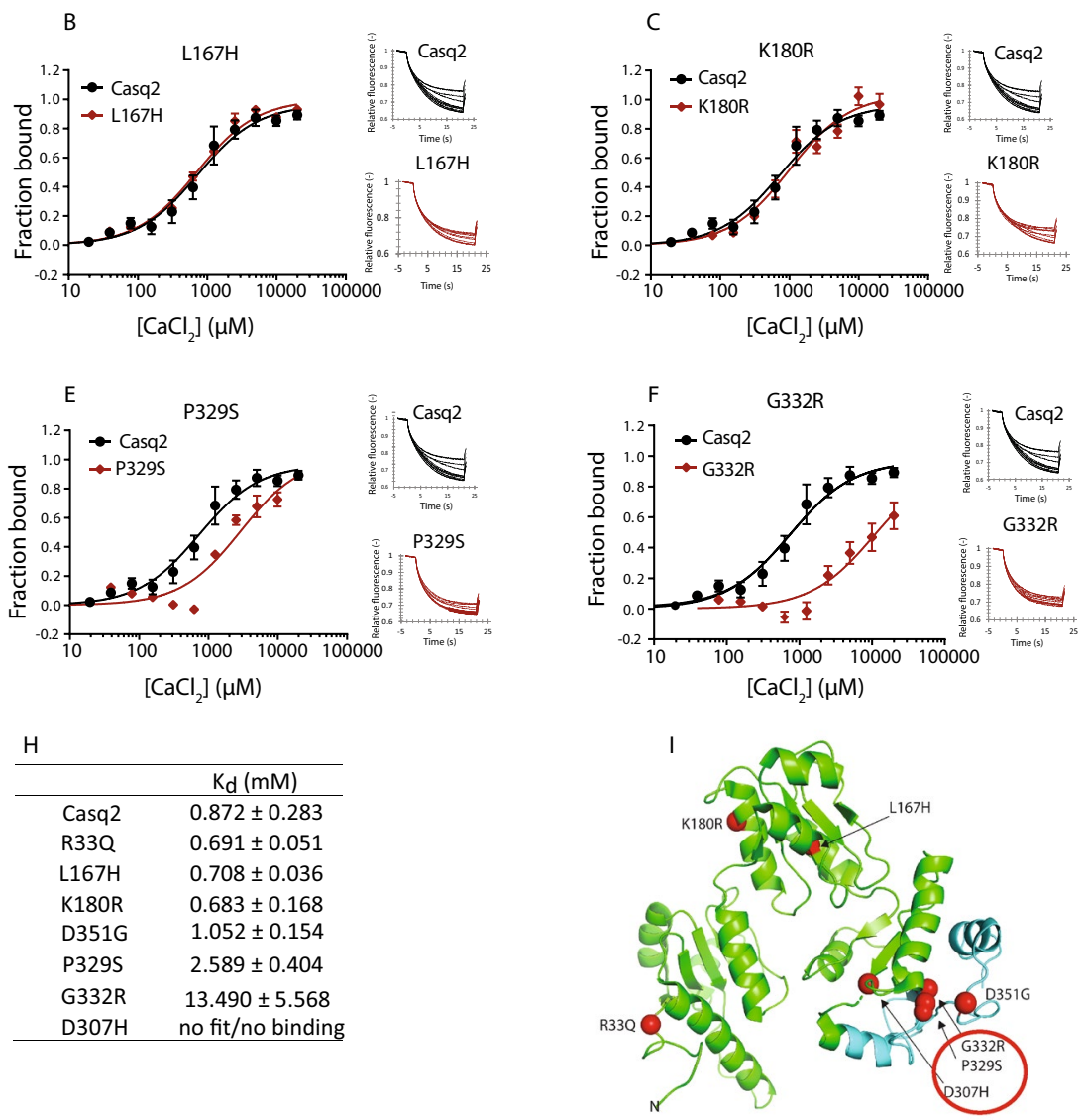

Figure 3. Calcium binding to calsequestrin mutants. (A-G) MST analysis of $\mathrm{Ca}^{2+}$ binding to mutants Casq2 (red line) and wild-type Casq2 (black line). (H) Calculated $\mathrm{Ca}^{2+}$ binding affinities of Casq2 mutants. (I) 3D structure of human Casq2 (adapted from 2VAF) with the location of mutants indicated in the Figure. The red circle depicts the location of mutations that affected $\mathrm{Ca} 2$ binding to Casq2.

Casq2 binding to IRE1 $\alpha$, an ER/SR stress sensor. Casq2 binds to IRE1 $\alpha$, an ER/SR stress sensor and squelches IRE1 $\alpha$ activity ${ }^{55}$. We used MST thermophoresis to test whether Casq2 mutations affected Casq2 interaction with the luminal domain of IRE1a. R33Q, L167H, D307H, P329S, G332R and D351G bound to the luminal domain of IRE1 a with similar kinetics and affinities as seen for wild-type protein (Fig. 9). However, the K180R mutant showed increased binding affinity (Fig. 9) indicating a stronger interaction between the K180R Casq2 mutant and the IRE1 $\alpha$ luminal domain. We concluded that all Casq2 mutants tested bound to the IRE1 $\alpha$ stress sensor.

\section{Discussion}

Our phylogenetic analysis of the calsequestrin genes (casq2, casq1, and pre-duplication casq) revealed that calsequestrin is an ancient protein within the metazoan, and duplication of the calsequestrin gene took place after the divergence of the lancelet but before divergence of Chondrichthyes. Duplication of the calsequestrin gene allowed for the differentiation of a muscle-specific form of the protein, namely cardiac calsequestrin (casq2) expressed in cardiomyocytes. In the heart $\mathrm{Ca}^{2+}$ release from the calsequestrin (Casq2) rich junctional SR is initiated by the $\mathrm{Ca}^{2+}$-induced $\mathrm{Ca}^{2+}$ release mechanism. In skeletal muscle, where Casq1 is expressed, $\mathrm{Ca}^{2+}$ release from the junctional SR is initiated by the depolarization-induced $\mathrm{Ca}^{2+}$ release mechanism. Notably in mammalian species Casq1 is almost exclusively expressed in skeletal muscle (Suppl. Fig. S3). However, Gallus gallus appears to have lost the Casq1 gene and Casq2 is highly expressed in both heart and skeletal muscle tissue (Suppl. Fig. S3). Other non-mammalian vertebrates also did not show the tissue-specific expression patterns of Casq1 vs 2 observed consistently in mammalian species suggesting that this is a later evolved mammalian-specific trait.

We also discovered that the Chondrichthye and Avian taxa lost casq1 but retained the casq2. Arthropoda do not have the calsequestrin gene, although they have transversely striated muscle similar to the vertebrate skeletal muscle ${ }^{56}$ with well-developed transverse tubules associated SR cisternae ${ }^{57,58}$. Considering that Arthropoda move by means of their segmental appendages, they may not require high capacity $\mathrm{Ca}^{2+}$ stores.

Comparison between the evolutionary distribution of calsequestrin and the RyR proteins ${ }^{58}$ provides insight into the evolution of the excitation-contraction coupling system in metazoan muscles. While we were unable to identify calsequestrin homologues, RyR homologues have been found in the genomes of holozoans such as 

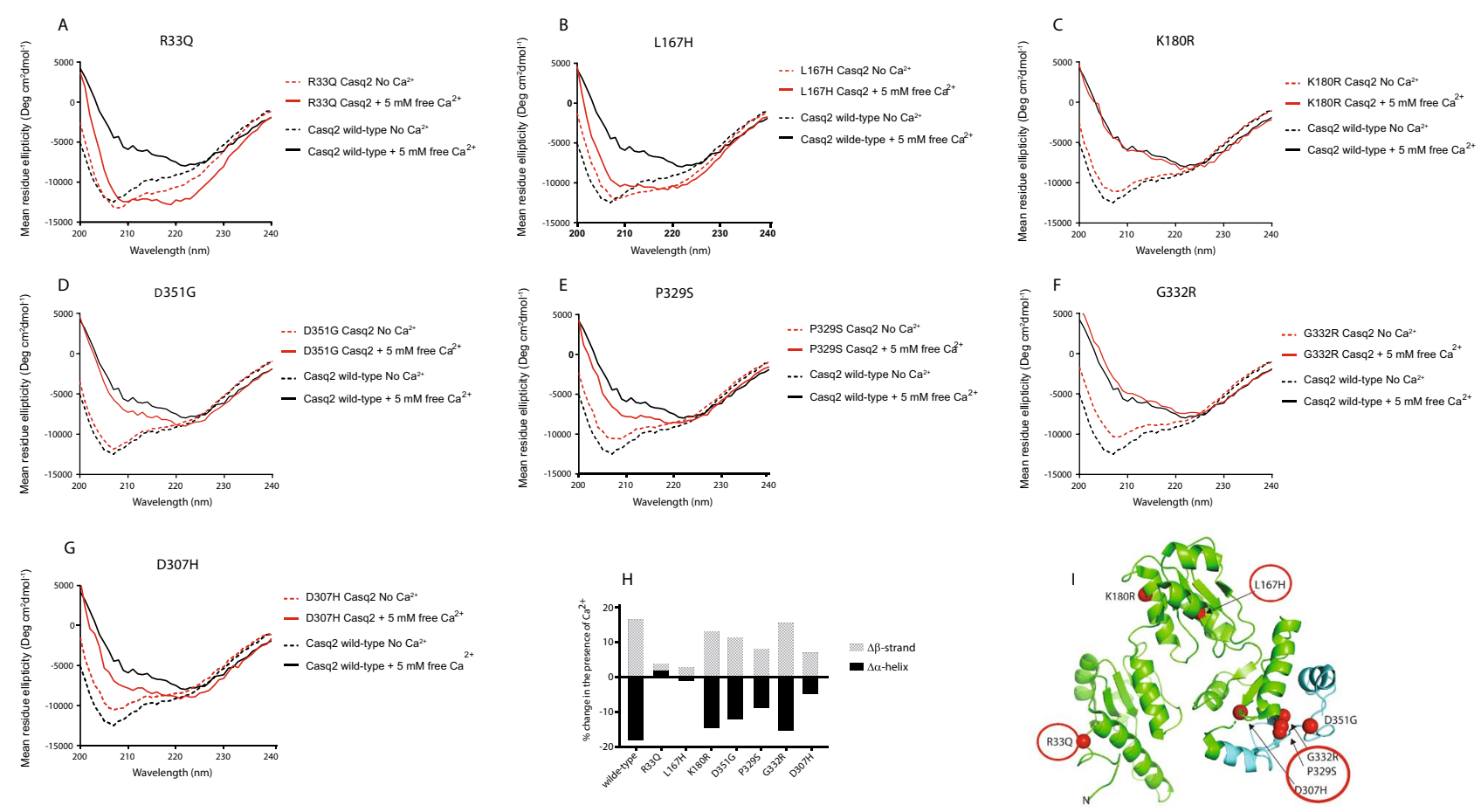

Figure 4. CD analysis of Casq2 mutants. (A-G) CD analysis of Casq 2 mutants (red solid line) and wild-type Casq2 (black solid line). $\mathrm{CD}$ analysis in the presence of $5 \mathrm{mM} \mathrm{Ca}^{2+}$ is indicted by black dotted lines for wild-type Casq 2 and by red dotted lines for Casq 2 mutants. (H) Change in $\%$ of $\alpha$-helix and $\beta$-strand content with the absence or presence of $\mathrm{Ca}^{2+}$ for wild-type Casq2 and each Casq2 mutant. Negative and positive values indicate a loss or gain of secondary structure content, respectively. (I) Three-dimensional structure of human Casq2 (2VAF). Red circles depict the location of mutations with altered CD spectrum.

\begin{tabular}{|c|c|c|c|c|c|c|c|c|}
\hline Casq2 & WT & R33Q & L167H & K180R & D307H & P329S & G332R & D351G \\
\hline \multicolumn{9}{|l|}{$\mathrm{No} \mathrm{Ca}^{2+}$} \\
\hline$\%$ a-helix & 26.18 & 28.83 & 26.71 & 23.7 & 24.055 & 21.36 & 20.37 & 24.31 \\
\hline$\% \beta$-sheet & 19.65 & 17.93 & 19.5 & 21.78 & 20.98 & 22.66 & 23.41 & 21.01 \\
\hline \multicolumn{9}{|l|}{$5 \mathrm{mM} \mathrm{Ca}^{2+}$} \\
\hline$\%$ a-helix & 7.88 & 30.78 & 25.48 & 8.95 & 19.485 & 12.17 & 4.86 & 11.88 \\
\hline$\% \beta$-sheet & 36.44 & 20.05 & 22.56 & 35.17 & 26.96 & 31.05 & 39.13 & 32.51 \\
\hline$\Delta a$-helix & -18.3 & 1.95 & -1.23 & -14.75 & -4.57 & -9.19 & -15.51 & -12.43 \\
\hline$\Delta \beta$-sheet & 16.79 & 2.12 & 3.06 & 13.39 & 5.98 & 8.39 & 15.72 & 11.5 \\
\hline
\end{tabular}

Table 1. CD analysis of calsequestrin mutants. WT, wild-type.

Capsaspora and choanoflagellates, suggesting that the RyR protein evolved first. The pairing of the calsequestrin and ryanodine receptors seems not to have been established as essential in non-vertebrate animals, given the lack of calsequestrin in sponges but presence in cniderians and ctenophores and the opposite for the RyRs ${ }^{58}$. The presence of the preduplicated RyR but absence of calsequestrin in insects suggests lack of essentiality of the paired regulation as well. However, both systems have been subject to expansions likely associated with two whole-genome duplication events near evolution of vertebrates ${ }^{58}$. Lineage-specific losses of the families are seen here as well in both cases. For example, amphibians lack the gene encoding RyR2, yet they express both forms of calsequestrin. These results support the notion of the appearance of multiple homologues of junctional SR proteins, including calsequestrin and $\mathrm{RyR}$, which are associated with depolarization-induced $\mathrm{Ca}^{2+}$ release or $\mathrm{Ca}^{2+}$-induced $\mathrm{Ca}^{2+}$ release mechanisms ${ }^{58}$.

Phylogenetic analysis of casq2 within the metazoan revealed a high level of conservation, especially in the beta-sheet in the hydrophobic core of the third thioredoxin-like fold. The C-terminal Asp rich domain of Casq2, responsible for high capacity low affinity $\mathrm{Ca}^{2+}$ binding, remained highly conserved throughout many different species. Of note, the pre-duplicated calsequestrin C-terminal domain, however, contains limited numbers of acidic residues, indicating a relatively low $\mathrm{Ca}^{2+}$ binding capacity in this basal lineage ${ }^{59}$. There are many highly conserved amino acid residues distributed throughout Casq2 that may be under evolutionary constraints, and 


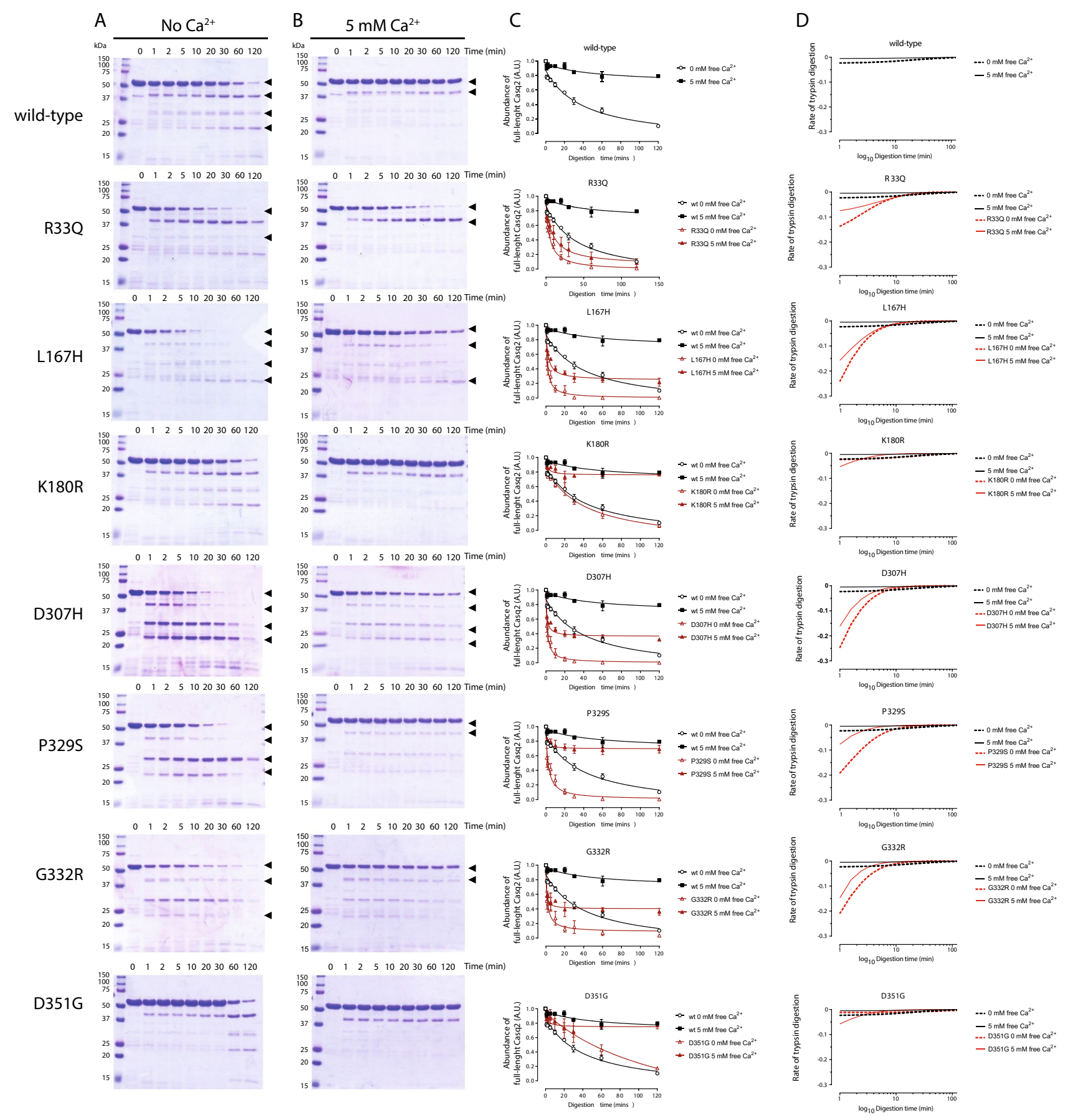

Figure 5. Limited trypsin digestion of Casq2 mutants. Wild-type Casq 2 and Casq 2 mutants were subjected to trypsin proteolysis in the absence (A) and presence $(\mathbf{B})$ of $5 \mathrm{mM} \mathrm{Ca}^{2+}$ followed by SDS-PAGE. The tryptic fragments, which differed from that of wild-type Casq2 are indicated by the arrowheads. (C) The rate of proteolysis of wild-type and mutant Casq2 as a function of time of trypsin digestion. (D) The rate of proteolysis plotted as the first derivative of the fitted curve from $(\mathbf{C})$.

mutations in these regions of the protein are expected to impact protein structure and function ${ }^{60,61}$. Not surprisingly, Casq2 mutations associated with CPVT are dispersed throughout different protein regions, but all are highly conserved throughout metazoans some (R33Q, K180R, D307H, P329S and G332R) even including preduplication Casq. Because of a specific disease phenotype of Casq2 mutants, one a priori prediction is that the mutations associated with CPVT would be in sites conserved in Casq2 but divergent in Casq1 and preduplicates, providing gain-of function amino acid changes in Casq2 which were then disrupted by the disease phenotypeassociated mutations. However, this was not what we observed. Instead we found strong conservation at these positions between the paralogues or indeed across all calsequestrin homologues. This suggests that the residues at these positions are critical for calsequestrin function. Specificity of cardiac disease seen with Casq2 mutants 

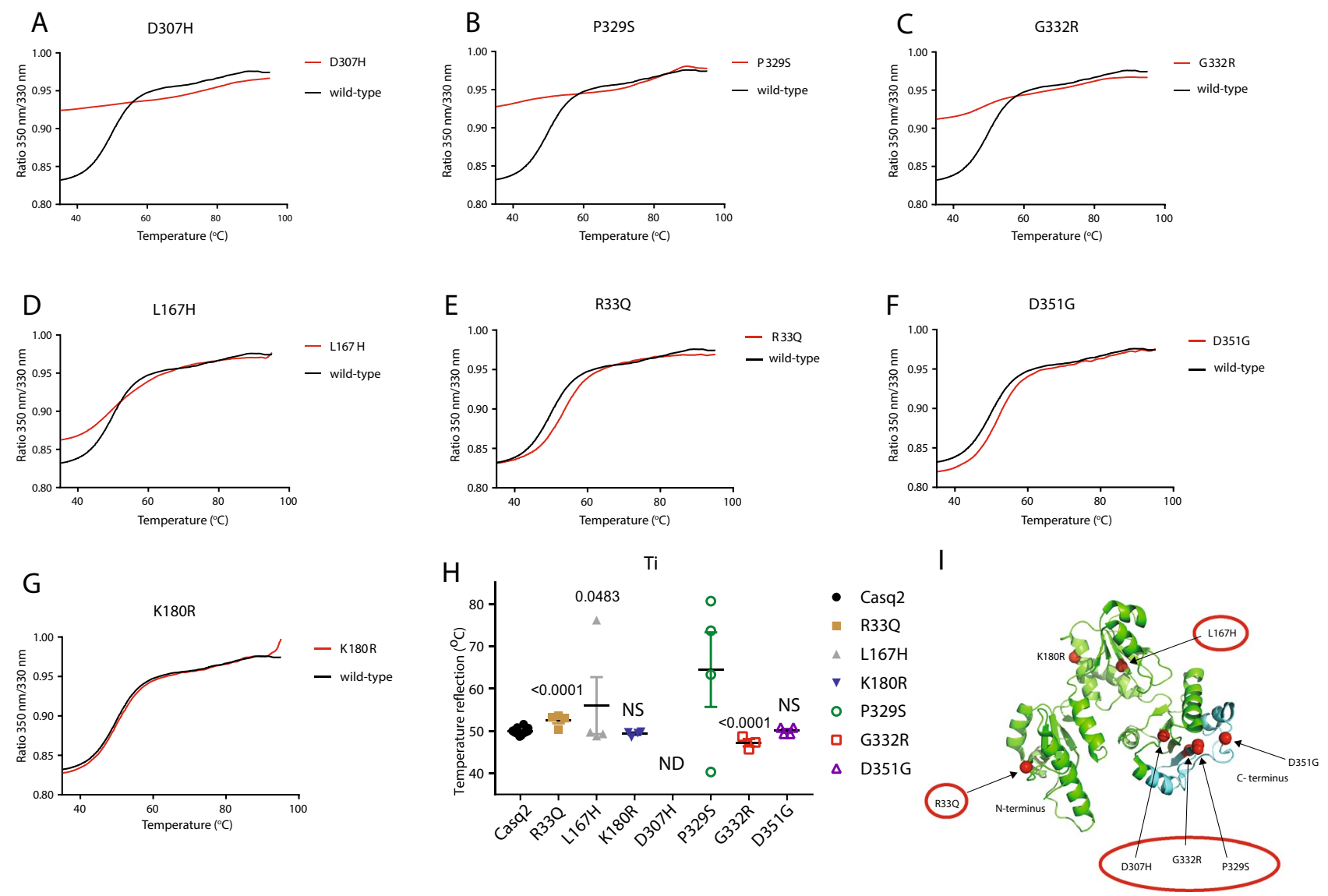

Figure 6. Thermal denaturation analysis of Casq 2 and Casq 2 mutants. (A-G) Thermal denaturation analysis of wild-type Casq2 (black lines) or Casq2 mutants (red lines) was monitored by intrinsic tryptophan fluorescence of proteins in response to increased temperature from 35 to $95^{\circ} \mathrm{C}$. Graphs represent 3 independent measurements. (H) Inflection temperature for Casq2 and Casq 2 mutants representing the temperature at which the transition from folded protein to unfolded state occurs. (I) Human Casq2 crystal structure (2VAF). Mutants with significant difference in their protein folding are indicated by red circles. The location of tryptophan residues are depicted as yellow sticks.

\begin{tabular}{|l|l|}
\hline Calsequestrin (Casq2) & Ti value \\
\hline Wild-type & $49.9 \pm 0.16(\mathrm{n}=18)$ \\
\hline R33Q & $52.5 \pm 0.73(\mathrm{n}=4)$ \\
\hline L167H & $56.0 \pm 6.73(\mathrm{n}=4)$ \\
\hline K180R & $49.4 \pm 0.29(\mathrm{n}=4)$ \\
\hline D351G & $50.0 \pm 0.43(\mathrm{n}=4)$ \\
\hline P329S & $64.5 \pm 8.82(\mathrm{n}=4)$ \\
\hline G332R & $47.2 \pm 0.87(\mathrm{n}=3)$ \\
\hline D307H & ND \\
\hline
\end{tabular}

Table 2. Ti values for Casq 2 and Casq2 mutants.

is likely due to the tissue-specific expression patterns of the paralogues. In humans there is little or no Casq1 paralogue expressed in cardiac tissue to compensate for Casq2 malfunction in CPVT.

For our biochemical studies, we have selected mutants linked to the human CPVT phenotype and located within highly conserved positions, namely, R33Q, L167H, K180R, D307H and three CASQ2 variants from whole-exome sequencing clinical testing, P329S, G332R, and D351G $\mathrm{G}^{24-27,39,46,62,63}$. In agreement with previous reports $^{25,30,34,36,53,64}$, our studies showed that Casq2 ${ }^{\mathrm{R} 33 \mathrm{Q}}$, Casq2 $2^{\mathrm{L} 167 \mathrm{H}}$, and Casq2 ${ }^{\mathrm{D} 307 \mathrm{H}}$ differed in their biochemical properties. Casq2 ${ }^{\mathrm{D} 307 \mathrm{H}}$ was reported to have significantly reduced $\mathrm{Ca}^{2+}$ binding capacity ${ }^{17}$. In this study, in label-free $\mathrm{MST} \mathrm{Ca}^{2+}$ binding assay the $\mathrm{D} 307 \mathrm{H}$ mutant showed no measurable $\mathrm{Ca}^{2+}$ binding and altered protein 
A
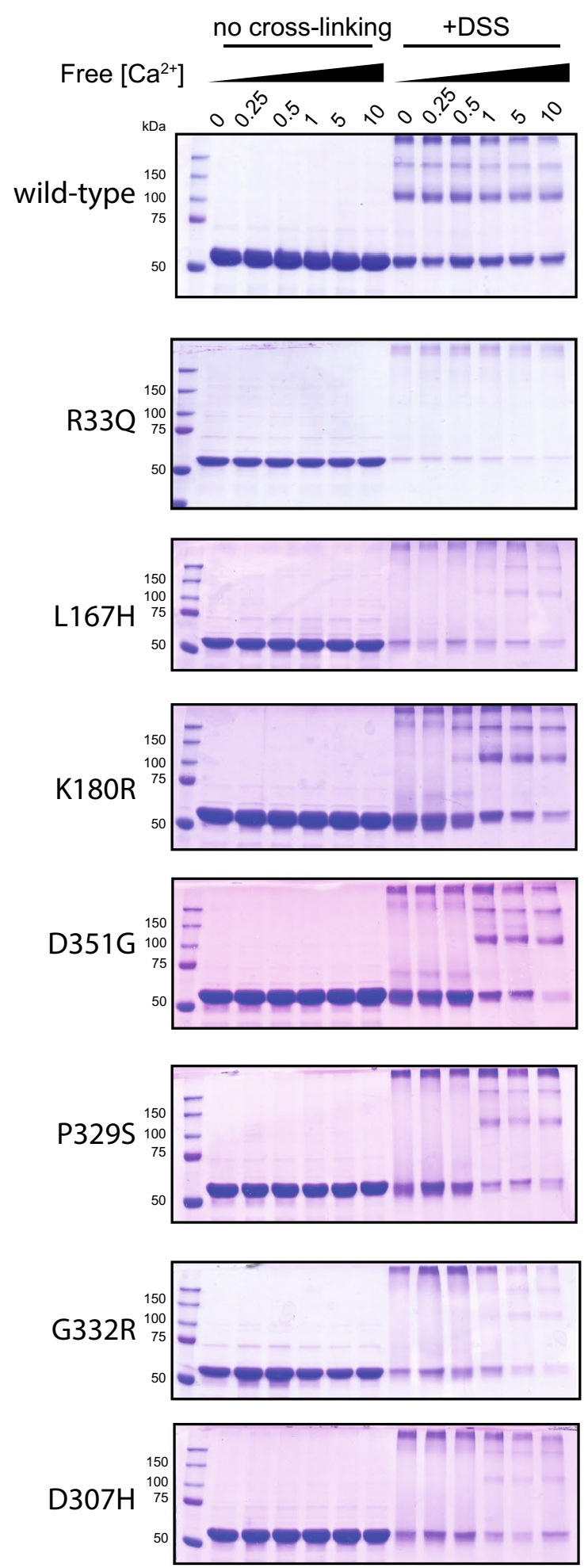

Coomassie Blue
B
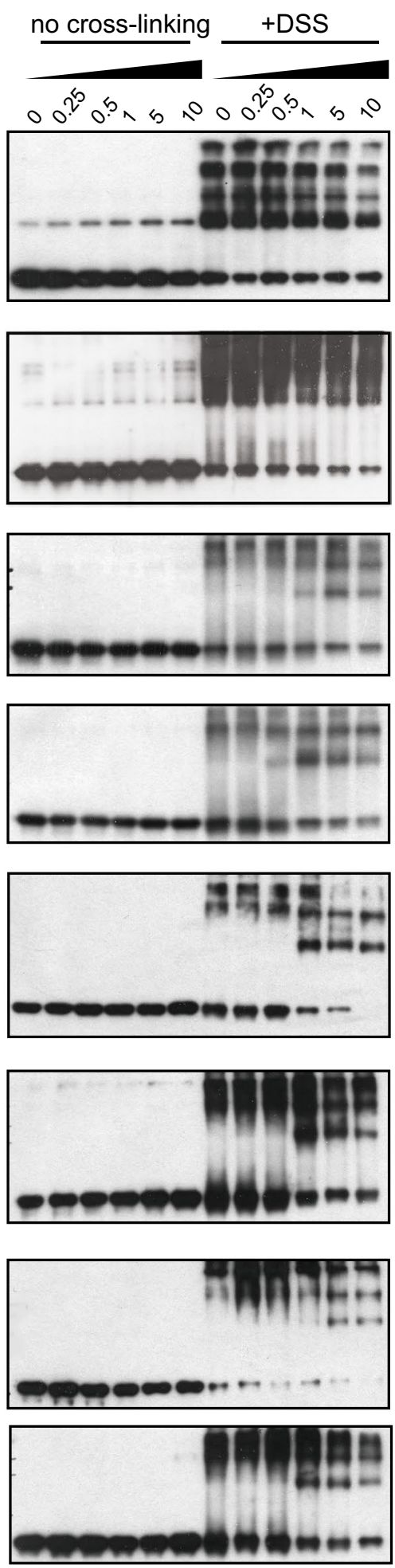

anti-Casq2 antibodies
C

Monomer band intensity in the presence of DSS cross-linker
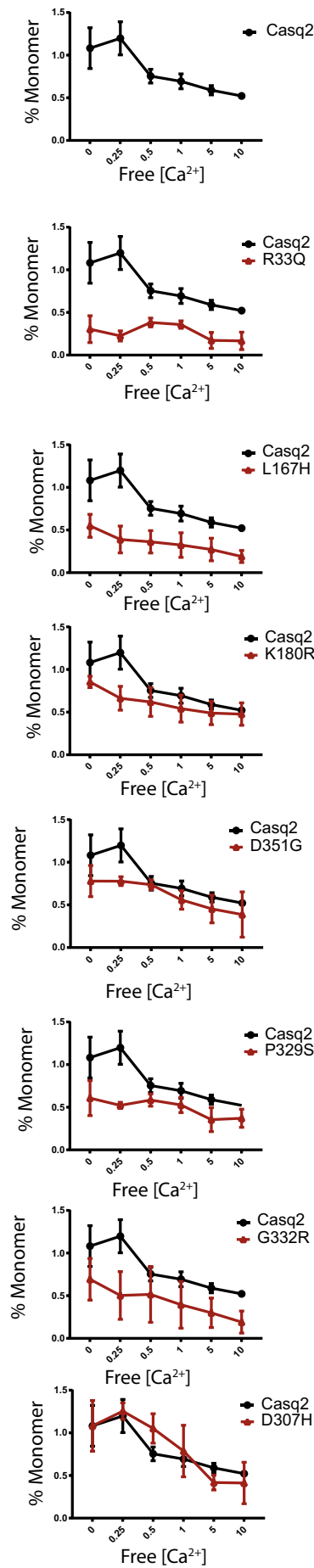

Free $\left[\mathrm{Ca}^{2+}\right]$

Figure 7. $\mathrm{Ca}^{2+}$-dependent polymerization of Casq2 and Casq2 mutants. (A) Coomassie blue stained SDSPAGE of Casq2 and Casq2 mutant incubated with or without cross-linker at increasing free $\mathrm{Ca}^{2+}$ concentration. (B) Immunoblots were probed with anti-Casq2 antibodies. The full-length blots are shown in Figs. S4 and S5. (C) Quantitative analysis of Casq2 monomer ( $\sim 50 \mathrm{kDa}$ protein band) of wild-type or mutant proteins in the presence of cross-linker (from $\mathbf{A}$ ) as a function of increased free $\mathrm{Ca}^{2}$ concentration. 
A

Coomassie blue staining

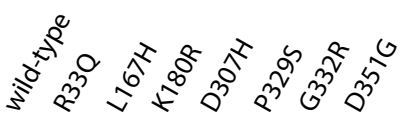

Native PAGE

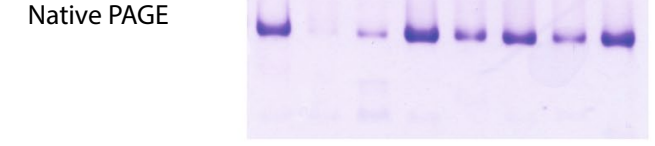

$\mathrm{kDa}$

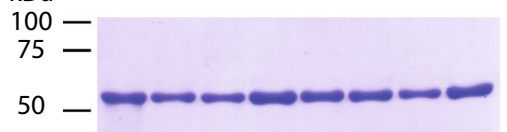

SDS-PAGE

B

Monomer band intensity

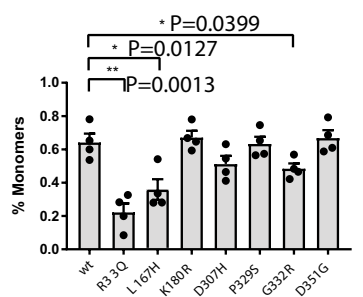

anti-Casq antibodies

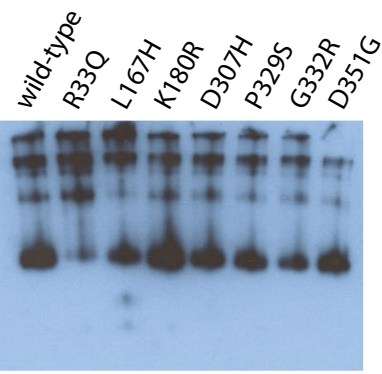

$\mathrm{kDa}$

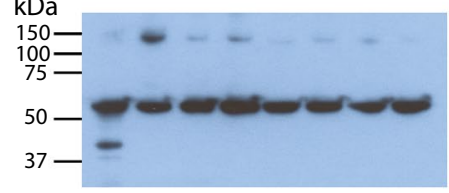

Dimer/Oligomers band intensity

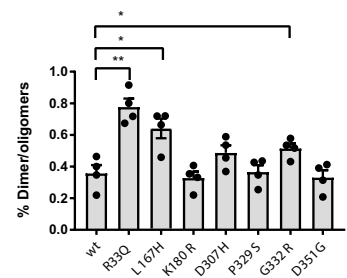

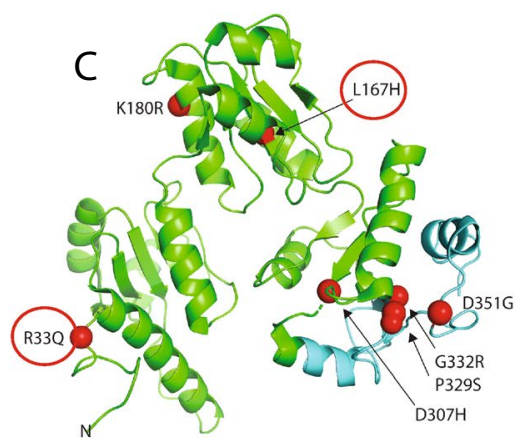

Figure 8. Polymerization of Casq 2 mutants. (A) Polymerization of Casq2 or Casq2 mutants was carried out at $167 \mu \mathrm{M}$ free $\mathrm{Ca}^{2}$ followed by SDS-PAGE or native gel electrophoresis. Immunoblots were probed with anit-Casq2 antibodies. A representative of four independent experiments is shown. The full-length blots are shown in Fig. S6. (B) Quantitative analysis of monomeric and oligomeric forms of Casq2 mutants. (C) Human Casq2 crystal structure (2VAF). Red circles depict the location of Casq2 mutations with highly increased oligomerization.

tertiary structure. R33Q and $\mathrm{L} 167 \mathrm{H}$ mutants retained $\mathrm{Ca}^{2+}$ binding capacity but had increased sensitivity to tryptic cleavage and lost $\mathrm{Ca}^{2+}$-dependent polymerization.

The Casq2 ${ }^{\mathrm{R} 33 \mathrm{Q}}$ and Casq2 ${ }^{\mathrm{L} 167 \mathrm{H}}$ mutants form large oligomers insensitive to $\mathrm{Ca}^{2+}$, indicating that they lost $\mathrm{Ca}^{2+}$ depend polymerization, and are not able to depolymerize in response to $\mathrm{Ca}^{2+}$ depletion, a critical function that affects the RyR2 channel gating response to depletion of $\mathrm{Ca}^{2+}$ during muscle contraction ${ }^{54}$. Amazingly, Casq2 $2^{\mathrm{D} 307 \mathrm{H}}$ substitution from aspartic acid to histidine, in the highly conserved hydrophobic core of third thioredoxin-like domain of Casq2, results in the loss of low affinity $\mathrm{Ca}^{2+}$ binding to Casq2. This is likely due to disruption of the third thioredoxin-like domain, a highly conserved region in Casq2. Surprisingly, Casq2 ${ }^{\mathrm{D} 307 \mathrm{H}}$ polymerized in a $\mathrm{Ca}^{2+}$-dependent manner, and, just like the wild-type Casq2, it exhibited $\mathrm{Ca}^{2+}$-dependent confirmation changes ${ }^{29,31}$. Nevertheless, the loss of low affinity and high capacity $\mathrm{Ca}^{2+}$ binding sites, due to severe Casq2 $2^{\mathrm{D} 307 \mathrm{H}}$ misfolding, results in reduced $\mathrm{Ca}^{2+}$ storage at the junctional SR, and impaired Casq $2 / \mathrm{Ca}^{2+}$-dependent regulation of RyR2 activity ${ }^{51,65}$. Animal studies revealed that in Casq2 ${ }^{\mathrm{D} 307 \mathrm{H} / \mathrm{D} 307 \mathrm{H}}$ knock-in mice there is a reduction in the abundance Casq2 $\mathrm{D} 307 \mathrm{H}$ protein ${ }^{29,66}$. Others reported no effect of $\mathrm{D} 307 \mathrm{H}$ mutation of protein stability in either animal model or in cultured cardiomyocytes ${ }^{31,33,51,65}$. Expression of Casq2 L167H mutant did not affect the level of the endogenous wild-type Casq2 ${ }^{34}$. However, in R33Q knock-in mice there was a significant reduction in the abundance of Casq2 $2^{\mathrm{R} 33 \mathrm{Q} / \mathrm{R} 33 \mathrm{Q}}$ protein ${ }^{30}$. This likely contributes to the CPVT phenotype.

K180R, P329S, G332R, and D351G mutants have not been previously studied with respect to their biochemical properties. K180R is a newly identified Casq2 mutant, and the first autosomal dominant mutant found of Casq2 ${ }^{27}$. Knollman's group recently reported a CPVT-like phenotype in a K180R heterozygous knock-in mouse model $^{46}$. Here we discovered that the Casq $2^{\mathrm{K} 180 \mathrm{R}}$ protein has indistinguishable biochemical properties from the wild-type Casq2, including $\mathrm{Ca}^{2+}$ binding affinity, secondary structure and conformation change in response to increased $\mathrm{Ca}^{2+}$ concentration, protein flexibility and conformation stability upon trypsin proteolysis, protein folding, and $\mathrm{Ca}^{2+}$ dependent polymerization. Recently, crystal structure studies of the Casq $2^{\mathrm{K} 180 \mathrm{R}}$ maps the mutation to the filament-forming interface ${ }^{67}$, and it was proposed that disrupted Casq 2 polymer formation may be responsible for Casq2 mutant-associated CPVT. Casq2 binds directly to the luminal domain of ER stress sensor IRE1 $\alpha$ at the junctional SR to prevent the activation of IRE $1 \alpha^{55}$. Interestingly, of all mutants tested in this 
A

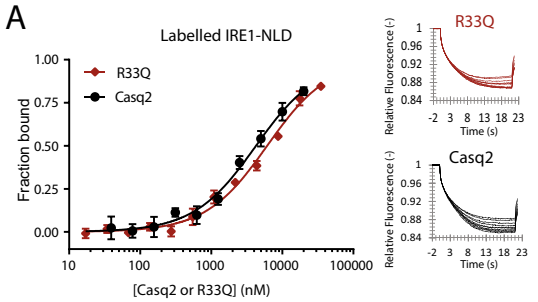

D Labelled IRE1-NLD

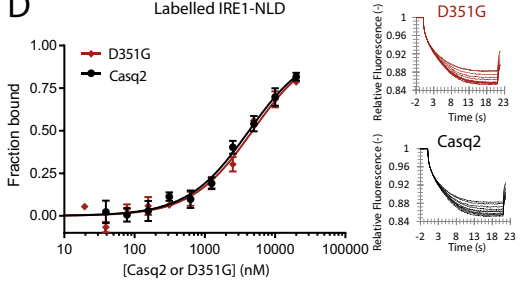

G

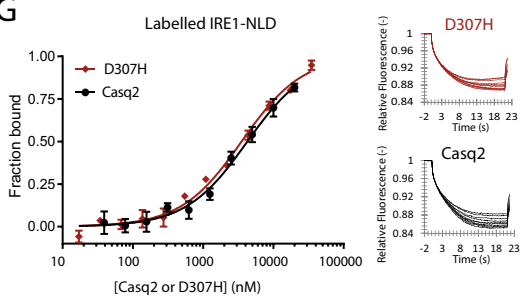

B

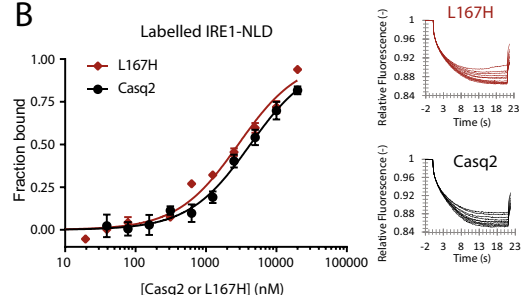

$\mathrm{E}$

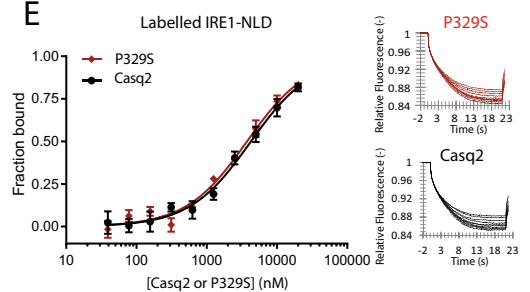

$\mathrm{H}$

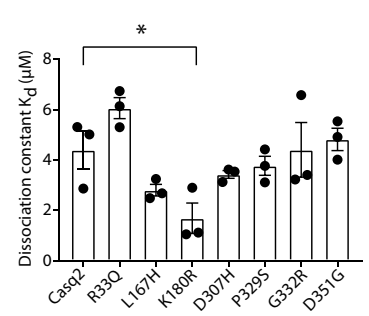

C

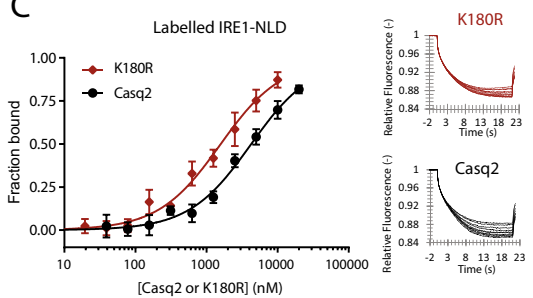

$\mathrm{F}$

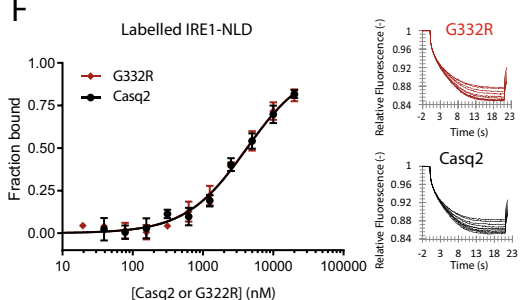

I

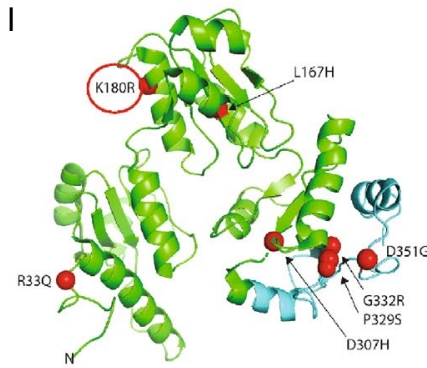

Figure 9. Casq2 mutants binding to the ER luminal domain of IRE1 $\alpha$. (A-G) Recombinant N-terminus luminal domain of IRE1a (IRE1-NLD) protein was covalently labeled with a red fluorescent tag and incubated with increasing amounts of Casq2 or Casq2 mutant as indicated in the Figure. Normalized MST time traces are shown to the right of the graph. Each data point is the average of three independent microscale thermophoresis measurements. (H) Bar graph depicts dissociation constants for different Casq2 mutants. ${ }^{*} \mathrm{p}=0.05$. (I) Human Casq2 crystal structure (2VAF). The red circle on the Casq2 3D structure depicts the location of mutation with altered IRE1-NLD binding.

study, only Casq $2^{\mathrm{K} 180 \mathrm{R}}$ showed altered binding to the luminal domain of IRE1 $\alpha$. Whether this is associated with CPVT remains to be established.

Casq2 ${ }^{\mathrm{D} 351 \mathrm{G} 39,42}$, Casq2 ${ }^{\mathrm{P} 329539}$ and Casq2 $2^{\mathrm{G} 332 \mathrm{R} 39}$ are three novel Casq2 variants ${ }^{39,42}$ located in a highly conserved third thioredoxin-like domain. Casq2 $2^{\mathrm{P} 329 \mathrm{~S}}$ and Casq2 ${ }^{\mathrm{G} 332 \mathrm{R}}$ have been identified as heterozygous carrier ${ }^{39}$. They are localized in the hydrophobic core of the highly conserved beta-sheet of the third thioredoxin-like domain and are highly conserved throughout the metazoan including Casq1 and pre-duplicate Casq. To our knowledge, there have been no reports on the biochemical and biophysical analysis of these mutants. Casq $2^{\mathrm{D} 351 \mathrm{G}}$ showed protein folding and function similar to wild-type protein, whereas Casq2 ${ }^{\mathrm{P} 329 \mathrm{~S}}$ and Casq2 $2^{\mathrm{G} 332 \mathrm{R}}$ showed similar properties to Casq $2^{\mathrm{D} 307 \mathrm{H}}$, including severe disruption in protein folding and impaired $\mathrm{Ca}^{2+}$ binding, indicative of an important structural and functional role for the highly conserved beta-sheet in the third thioredoxin-like domain of Casq2.

Overall, the three CPVT disease causing Casq2 mutants (R33Q, L167H, D307H) and two heterozygous variants (P329S and G332R) may lead to CPVT via different mechanisms. The third thioredoxin-like fold domain contains one highly conserved beta-sheet consist of four beta-strands, which are essential for correct folding and $\mathrm{Ca}^{2+}$ binding, mutations in this region including D307H, P329S, and G332R, and all result in severely misfolded protein with reduced or lost $\mathrm{Ca}^{2+}$ binding. $\mathrm{R} 33 \mathrm{Q}, \mathrm{L} 167 \mathrm{H}$ and $\mathrm{K} 180 \mathrm{R}$ are located at the Casq2 front-to-front and back-to-back polymerization interface, causing dysfunction in the protein's $\mathrm{Ca}^{2+}$ depend polymerization/ depolymerization that would affect filament formation, as proposed by Titus et al. ${ }^{67}$. This may be the unifying feature of Casq2 mutants association with $\mathrm{CPVT}^{67}$.

\section{Experimental procedures}

Genome databases. The genomes used in the comparative genomics and phylogenetics analyses are publicly available and include the following from NCBI: Homo sapiens, Canis lupus familiaris, Bos taurus, Oryctolagus cuniculus, Sus scrofa, Rattus norvegicus, Mus musculus, Xenopus tropicalis, Gallus gallus, Taeniopygia guttata, Crocodylus porosus, Latimeria chalumnae, Danio rerio, Callorhinchus milii, Leucoraja erinacea, Branchiostoma floridae, Ciona intestinalis, Helobdella robusta, Drosophila melanogaster, Apis mellifera, Manduca sexta, Daphnia pulex, Parhyale hawaiensis, Parasteatoda tepidariorum, Caenorhabditis elegans, Nematostella vectensis, Mnemi- 
opsis leidyi, Trichoplax adhaerans, Amphimedon queenslandica, Monosiga brevicollis, Salpingoeca rosetta, Capsaspora owczarzaki. Ensembl: Helobdella robusta. Skatebase.org: Leucoraja erinacea. hymenopteragenome.org: Apis mellifera. wfleabase.org: Daphnia pulex.

Comparative genomics, phylogenetic and sequence alignments. Using $H$. sapiens CASQ1 and CASQ2 sequences as queries, BLASTp (Basic Local Alignment Search Tool protein) searches were performed on the genomes of 28 metazoan organisms (Supplementary Table S1). Reciprocal BLAST was performed to verify the homology of significant hits obtained via forward BLAST. Predictions regarding the homology of a sequence were based on both the E-value and identity score. Hits that displayed the lowest E-value and greatest identity score in both the forward and reciprocal BLAST were predicted as being homologous. In cases of multiple homologous hits, the hit with the greatest identity score was predicted as being potentially orthologous. When no significant hits could be obtained using a BLASTp search, tBLASTn was used to search inside of the genome scaffolds. Additionally, HMMer was also used to search for sequences without significant BLASTp hits. Any potential HMMer hits were then verified using reciprocal BLAST.

For phylogenetic analyses, we used both RAxML consensus trees using 100 bootstraps and MrBayes Bayesian analysis trees with 10 million iterations achieving an average standard deviation of splits frequencies value of less than 0.01. Default parameters on CIPRES for RAxML-HPC v0.8 and MrBayes v3.2.6 on XSEDE were used with the following change for the RAxML trees: an LG4X protein matrix was used with the PROTGAMMA protein substitution model. The clades generated by the RAxML consensus trees were considered significant with node values of at least 50. MrBayes tree clades were considered significant with node probability values of at least 0.8. RAxML consensus trees were generated using Consensus v3.695, while the graphical representation of the phylogenetic results was generated using Figtree v1.3.1. The calsequestrin sequences found using comparative genomics were aligned using MUSCLE v3.8.31 and visualized using MESQUITE v3.2.

Site-directed mutagenesis. A pET22b E. coli expression vector containing full-length recombinant canine casq2 cDNA with a C-terminus 6 xHis tag were used as template to obtain Casq2 mutants. Platinum pfx DNA polymerase (Invitrogen, 11708) was used for site-directed mutagenesis PCR with primers (Supp. Table S2). Methylated non-mutated template plasmids were digested with DpnI, and the correct mutations were confirmed by DNA sequencing. Seven CPVT-related Casq2 mutants (R33Q, L167H, K180R, D307H, P329S, G332R, D351G) were generated.

Protein purification. cDNA encoding wild-type canine cardiac casq2 and casq2 mutants was cloned into pET22b expression vector. Proteins were expressed in E. coli BL21(DE3) and purified. Cells were grown in LB medium until the $\mathrm{A}_{600}$ reaches 0.6 at $37^{\circ} \mathrm{C}$, then induced with $1 \mathrm{mM}$ IPTG at $37^{\circ} \mathrm{C}$ for $3 \mathrm{~h}$. Cells were crashed into a buffer containing $50 \mathrm{mM}$ Tris- $\mathrm{HCl}, \mathrm{pH} 8.0,300 \mathrm{mM} \mathrm{NaCl}$, and $10 \%$ glycerol, then purified by using a HisTrap HP purification column (GE lifesciences, 17524701) and AKTA pure chromatography system (GE lifesciences 29018224). Purification was performed using binding buffer containing $50 \mathrm{mM}$ Tris- $\mathrm{HCl}, \mathrm{pH} 8.0$, $300 \mathrm{mM} \mathrm{NaCl}$, and protein eluted with buffer containing $50 \mathrm{mM}$ Tris- $\mathrm{HCl}, \mathrm{pH} 8.0,300 \mathrm{mM} \mathrm{NaCl}$, and $250 \mathrm{mM}$ imidazole. The 6 xHis tagged ER luminal domain of IRE1 a (IRE1-NLD) was expressed in COS-1 cells and purified by Ni-NTA agarose chromatography ${ }^{55}$. Protein concentration was determined using Bio-Rad $D C$ protein assay (Bio-Rad, 5000111) as recommended by the manufacturer. Protein concentration was also determined by measuring A280 absorbance using Nanovue plus spectrophotometer (GE Healthcare).

CD analysis. CD spectra were recorded on a Jasco model J-810 spectropolarimeter. Far UV CD spectra were collected with $4.82 \mu \mathrm{M}$ protein in buffer containing $10 \mathrm{mM} \mathrm{NaH}_{2} \mathrm{PO}_{4}, \mathrm{pH} 7.4$, and $5 \mathrm{mM} \mathrm{KCl}$, as $\mathrm{NaCl}$ interferes with CD analysis. CD scans were recorded using a quartz cell with a path length of $1 \mathrm{~mm}$, response time of $2 \mathrm{~s}$, scan speed of $10 \mathrm{~nm} / \mathrm{min}$, and band width of $1.0 \mathrm{~nm}$. $\mathrm{Ca}^{2+}$-induced changes in CD spectra were monitored in the presence of $1 \mathrm{mM}$ of EGTA and $6 \mathrm{mM}$ of $\mathrm{CaCl}_{2}$. $\mathrm{CD}$ spectra analysis was carried out at $24^{\circ} \mathrm{C}$. The final spectra was an average of 5 measurements, after baseline subtraction. Analysis of the spectra was performed using $\mathrm{K} 2 \mathrm{D} 3{ }^{68}$. The following calculations were performed for analysis:

Mean residue ellipticity was calculated with formula:

$$
[\theta]=\theta_{o b s} \times \frac{M R W}{(10 \times l \times c)}
$$

where $[\theta]$ with unit of Deg $\mathrm{cm}^{2} \mathrm{dmol}^{-1}, \theta_{\text {obs }}$ is the observed ellipticity in degrees, $l$ is the optical path-length in $\mathrm{cm}, c$ is the protein concentration in $\mathrm{g} / \mathrm{ml}$, MRW is the mean residue molecular mass calculated with formula:

$$
M R W=\frac{M}{(N-1)}
$$

where $M$ is a molecular mass of polypeptide chain in $\mathrm{Da}$, and $N$ is the number of amino acid in the chain.

MST and thermal denaturation analyses. Labelled MST. MST analyses were carried out using a Monolith NT.115 instrument (Nano Temper Technologies, Germany) or Monolith NT.LabelFree instrument (Nano Temper Technologies, Germany). The ER-luminal domain of IRE1a (IRE1-NLD) was labelled using the Monolith NT Protein Labeling Kit RED-NHS (Nano Temper Technologies, cat\# MO-C030) following the manufacture's protocol. All experiments were carried out at room temperature in standard capillaries with $20 \%$ 
LED power (fluorescence lamp intensity) and 40\% MST power (IR-laser intensity). The assay buffer contained $50 \mathrm{mM}$ HEPES, pH 7.4, $150 \mathrm{mM} \mathrm{KCl}, 500 \mu \mathrm{M} \mathrm{CaCl}_{2}, 250 \mu \mathrm{M}$ EGTA, 0.05\% Tween-20, and 2.5\% glycerol. $\mathrm{CaCl}_{2}$ and EGTA concentrations were adjusted to obtain the desired free $\mathrm{Ca}^{2+}$ concentration: no $\mathrm{Ca}^{2+}(500 \mu \mathrm{M}$ EGTA, $\left.500 \mu \mathrm{M} \mathrm{CaCl}_{2}\right), 5 \mathrm{mM}\left(500 \mu \mathrm{M}\right.$ EGTA, $\left.5.5 \mathrm{mM} \mathrm{CaCl}_{2}\right)$. Free $\mathrm{Ca}^{2+}$ concentration was calculated using the $\mathrm{Ca}^{2+}$-EGTA Calculator TS v1.3 web tool ${ }^{69}$.

Label-free MST. $\mathrm{Ca}^{2+}$ binding to wild-type Casq2 or Casq2 mutants were carried out using a Monolith NT.LabelFree instrument in standard capillaries with $20 \%$ LED power and $60 \%$ MST power. The proteins were incubated for $10 \mathrm{~min}$ in a buffer containing $50 \mathrm{mM}$ HEPES, pH 7.4, $150 \mathrm{mM} \mathrm{KCl}, 0.1 \%$ pluronic F-127, and $50 \mu \mathrm{M}$ EGTA. An increasing concentration of $\mathrm{CaCl}_{2}(0.01-20 \mathrm{mM}$, in $50 \mathrm{mM}$ HEPES, pH 7.4, $150 \mathrm{mM} \mathrm{KCl})$ was used. All MST data was analyzed by Monolith Affinity Analysis v2.2.6 software.

Tycho NT.6. Thermal denaturation analysis of wild-type Casq2 or Casq2 mutants was carried out using Tycho NT.6. This label-free system is based on measurement of a protein's intrinsic tryptophan fluorescence and records a protein's unfolding profile in real-time as temperature is increased from 35 to $95^{\circ} \mathrm{C}$. Ten $\mu \mathrm{lof} 0.25 \mathrm{mg} /$ $\mathrm{ml}$ protein was used in buffer containing $50 \mathrm{mM}$ HEPES, pH 7.4, $150 \mathrm{mM} \mathrm{KCl}, 500 \mu \mathrm{M} \mathrm{CaCl}_{2}, 250 \mu \mathrm{M} \mathrm{EGTA}$, $0.05 \%$ Tween-20, and $2.5 \%$ glycerol.

Native polyacrylamide gel electrophoresis. To determining the oligomerization state of Casq2 or Casq2 mutants in the presence of the same free $\mathrm{Ca}^{2+}$ concentration $(167 \mu \mathrm{M})$, a discontinuous Tris-glycine polyacrylamide gel system consisting of a $5 \%$ stacking gel and a $10 \%$ separation gel was used under non-denaturing conditions. Proteins were diluted $3 \times$ with non-denaturing loading dye $(240 \mathrm{mM}$ Tris- $\mathrm{HCl}, \mathrm{pH} 6.8,30 \%$ glycerol, and $0.03 \%$ bromophenol blue). Proteins were separated in a Mini-PROTEAN electrophoresis chamber (BioRad) in a running buffer containing $25 \mathrm{mM}$ Tris, $\mathrm{pH} 8.8$, and $192 \mathrm{mM}$ glycine, at $100 \mathrm{~V}$, for $2 \mathrm{~h}$ at $4{ }^{\circ} \mathrm{C}$. The proteins were stained with Stains-all solution ${ }^{70}$, Coomassie-blue, or transferred to nitrocellulose membrane for immunoblotting analysis.

Limited proteolysis. Cardiac calsequestrin (Casq2) and mutant proteins were subjected to proteolysis in a buffer containing $50 \mathrm{mM}$ HEPES, pH 7.4, $150 \mathrm{mM} \mathrm{KCl}, 500 \mu \mathrm{M} \mathrm{CaCl}_{2}, 250 \mu \mathrm{M} \mathrm{EGTA}, 0.05 \%$ Tween-20, and $2.5 \%$ glycerol. $\mathrm{CaCl}_{2}$ and EGTA concentrations were adjusted to the desired free $\mathrm{Ca}^{2+}$ concentration: no $\mathrm{Ca}^{2+}$ $\left(250 \mu \mathrm{M}\right.$ EGTA, $\left.250 \mu \mathrm{M} \mathrm{CaCl}_{2}\right), 5 \mathrm{mM}\left(250 \mu \mathrm{M}\right.$ EGTA, $\left.5.25 \mathrm{mM} \mathrm{CaCl}_{2}\right)$. Free $\mathrm{Ca}^{2+}$ concentration was calculated using the Ca-EGTA Calculator TS v1.3 web tool ${ }^{69}$. Proteins $(150 \mu \mathrm{g}$ of protein in $200 \mu \mathrm{l})$ were incubated in a reaction buffer with the desired free $\mathrm{Ca}^{2+}$ concentration for 20 min at $25{ }^{\circ} \mathrm{C}$ before addition of trypsin at the trypsin/protein ration of 1:50 (w/w), and samples were taken for SDS-PAGE analysis at 1,2,5, 10, 20, 30, 60, and $120 \mathrm{~min}$. The samples were mixed with $4 \times$ SDS-PAGE sample buffer (Bio-Rad) containing PMSF, and boiled at $100^{\circ} \mathrm{C}$ for $2 \mathrm{~min}$ before SDS-PAGE. All experiments were repeated 3 times with protein from 2 separate purifications. The gels were stained with Coomassie Brilliant Blue R-250 (Bio-Rad).

Cross-linking. The homobiofunctional protein cross linker disuccinimidyl suberate (DSS) (Thermo Scientific Pierce, cat\#:21555) was dissolved in DMSO at a final concentration of $10 \mathrm{mM}^{55}$. Protein concentration of wild-type and Casq2 mutant proteins was determined using Bio-Rad DC protein assay and the proteins were diluted to a final concentration of $10 \mu \mathrm{M}$ in a reaction buffer containing $50 \mathrm{mM} \mathrm{HEPES}, \mathrm{pH} 7.4,150 \mathrm{mM} \mathrm{NaCl}$, $250 \mu \mathrm{M}$ EGTA, $500 \mu \mathrm{M} \mathrm{CaCl}_{2}$, and $0.05 \%$ Tween-20. Proteins were incubated with 20 -fold molar excess of DSS for $1 \mathrm{~h}$ at $22.5^{\circ} \mathrm{C}$. The reaction was then quenched for $15 \mathrm{~min}$ with $100 \mathrm{mM}$ Tris $\mathrm{pH} 7.4$ followed by SDS-PAGE ( $10 \%$ acrylamide). Proteins were transferred to nitrocellulose membrane follow by immunoblotting with mouse anti-6xHis antibodies (ThermoFisher, MA1-21315) or anti-calsequestrin antibodies (abcam, 3516).

Statistical analysis. Statistical analysis was performed using GraphPad Prism version 7.0. The Student's t-test was used to compare the mean of two independent groups, and one-way Anova was used to compare the mean of three or more independent groups, with a $p$-value determined to be significant if less than 0.05 .

Received: 12 June 2020; Accepted: 8 October 2020

Published online: 22 October 2020

\section{References}

1. Rossi, A. E. \& Dirksen, R. T. Sarcoplasmic reticulum: The dynamic calcium governor of muscle. Muscle Nerve. 33(6), 715-731. https://doi.org/10.1002/mus.20512 (2006) (Epub 2006/02/16).

2. Eisner, D. A., Caldwell, J. L., Kistamas, K. \& Trafford, A. W. Calcium and excitation-contraction coupling in the heart. Circ. Res. 121(2), 181-195. https://doi.org/10.1161/CIRCRESAHA.117.310230 (2017).

3. Reddish, F. N., Miller, C. L., Gorkhali, R. \& Yang, J. J. Calcium dynamics mediated by the endoplasmic/sarcoplasmic reticulum and related diseases. Int. J. Mol. Sci. 18(5), 1024. https://doi.org/10.3390/ijms18051024 (2017) (Epub 2017/05/11).

4. Michalak, M. \& Opas, M. Endoplasmic and sarcoplasmic reticulum in the heart. Trends Cell Biol. 19(6), 253-259. https://doi. org/10.1016/j.tcb.2009.03.006 (2009) (Epub 2009/05/05).

5. Wray, S. \& Burdyga, T. Sarcoplasmic reticulum function in smooth muscle. Physiol. Rev. 90(1), 113-178. https://doi.org/10.1152/ physrev.00018.2008. (2010) (Epub 2010/01/21).

6. Bers, D. M. Cardiac sarcoplasmic reticulum calcium leak: Basis and roles in cardiac dysfunction. Annu. Rev. Physiol. 76, 107-127. https://doi.org/10.1146/annurev-physiol-020911-153308 (2014). 
7. Michalak, M. \& Agellon, L. B. Stress coping strategies in the heart: An integrated view. Front. Cardiovasc. Med. 5, 168. https://doi. org/10.3389/fcvm.2018.00168. (2018) (Epub 2018/12/07).

8. Barone, V., Randazzo, D., Del Re, V., Sorrentino, V. \& Rossi, D. Organization of junctional sarcoplasmic reticulum proteins in skeletal muscle fibers. J. Muscle Res. Cell Motil. 36(6), 501-515. https://doi.org/10.1007/s10974-015-9421-5 (2015).

9. Chopra, N. \& Knollmann, B. C. Triadin regulates cardiac muscle couplon structure and microdomain $\mathrm{Ca}(2+)$ signalling: A path towards ventricular arrhythmias. Cardiovasc. Res. 98(2), 187-191. https://doi.org/10.1093/cvr/cvt023. (2013) (Epub 2013/02/12).

10. Costello, B. et al. Characterization of the junctional face membrane from terminal cisternae of sarcoplasmic reticulum. J. Cell Biol. 103(3), 741-753 (1986) (Epub 1986/09/01).

11. Lee, J. M. et al. Negatively charged amino acids within the intraluminal loop of ryanodine receptor are involved in the interaction with triadin. J. Biol. Chem. 279(8), 6994-7000. https://doi.org/10.1074/jbc.M312446200 (2004) (Epub 2003/11/26).

12. Kobayashi, Y. M., Alseikhan, B. A. \& Jones, L. R. Localization and characterization of the calsequestrin-binding domain of triadin. 1 Evidence for a charged beta-strand in mediating the protein-protein interaction. J. Biol. Chem. 275(23), 17639-17646. https:// doi.org/10.1074/jbc.M002091200 (2000) (Epub 2000/04/05).

13. Gyorke, I., Hester, N., Jones, L. R. \& Gyorke, S. The role of calsequestrin, triadin, and junctin in conferring cardiac ryanodine receptor responsiveness to luminal calcium. Biophys. J. 86(4), 2121-2128. https://doi.org/10.1016/S0006-3495(04)74271-X. (2004) (Epub 2004/03/26).

14. Knollmann, B. C. New roles of calsequestrin and triadin in cardiac muscle. J. Physiol. 587(Pt 13), 3081-3087. https://doi. org/10.1113/jphysiol.2009.172098. (2009) (Epub 2009/05/20).

15. Gyorke, S., Stevens, S. C. \& Terentyev, D. Cardiac calsequestrin: Quest inside the SR. J. Physiol. 587(Pt 13), 3091-3094. https://doi. org/10.1113/jphysiol.2009.172049. (2009) (Epub 2009/07/02).

16. Wang, S. et al. Crystal structure of calsequestrin from rabbit skeletal muscle sarcoplasmic reticulum. Nat. Struct. Biol. 5(6), 476-483 (1998).

17. Kim, E. et al. Characterization of human cardiac calsequestrin and its deleterious mutants. J. Mol. Biol. 373(4), 1047-1057. https ://doi.org/10.1016/j.jmb.2007.08.055. (2007) (Epub 2007/09/21).

18. Baltogiannis, G. G. et al. CPVT: Arrhythmogenesis, therapeutic management, and future perspectives. A brief review of the literature. Front. Cardiovasc. Med. 6, 92. https://doi.org/10.3389/fcvm.2019.00092. (2019) (Epub 2019/08/06).

19. Mohamed, U., Napolitano, C. \& Priori, S. G. Molecular and electrophysiological bases of catecholaminergic polymorphic ventricular tachycardia. J. Cardiovasc. Electrophysiol. 18(7), 791-797. https://doi.org/10.1111/j.1540-8167.2007.00766.x. (2007) (Epub 2007/06/21).

20. Priori, S. G. et al. Clinical and molecular characterization of patients with catecholaminergic polymorphic ventricular tachycardia. Circulation 106(1), 69-74. https://doi.org/10.1161/01.cir.0000020013.73106.d8. (2002) (Epub 2002/07/03).

21. Postma, A. V. et al. Absence of calsequestrin 2 causes severe forms of catecholaminergic polymorphic ventricular tachycardia. Circ. Res. 91(8), e21-e26. https://doi.org/10.1161/01.res.0000038886.18992.6b. (2002) (Epub 2002/10/19).

22. Nyegaard, M. et al. Mutations in calmodulin cause ventricular tachycardia and sudden cardiac death. Am. J. Hum. Genet. 91(4), 703-712. https://doi.org/10.1016/j.ajhg.2012.08.015. (2012) (Epub 2012/10/09).

23. Roux-Buisson, N. et al. Absence of triadin, a protein of the calcium release complex, is responsible for cardiac arrhythmia with sudden death in human. Hum. Mol. Genet. 21(12), 2759-2767. https://doi.org/10.1093/hmg/dds104. (2012) (Epub 2012/03/17).

24. Faggioni, M., Kryshtal, D. O. \& Knollmann, B. C. Calsequestrin mutations and catecholaminergic polymorphic ventricular tachycardia. Pediatr. Cardiol. 33(6), 959-967. https://doi.org/10.1007/s00246-012-0256-1. (2012) (Epub 2012/03/17).

25. Terentyev, D. et al. Abnormal interactions of calsequestrin with the ryanodine receptor calcium release channel complex linked to exercise-induced sudden cardiac death. Circ. Res. 98(9), 1151-1158. https://doi.org/10.1161/01.RES.0000220647.93982.08. (2006) (Epub 2006/04/08).

26. Terentyev, D. et al. Modulation of SR Ca release by luminal Ca and calsequestrin in cardiac myocytes: Effects of CASQ2 mutations linked to sudden cardiac death. Biophys. J. 95(4), 2037-2048. https://doi.org/10.1529/biophysj.107.128249. (2008) (Epub 2008/05/13).

27. Gray, B. et al. A novel heterozygous mutation in cardiac calsequestrin causes autosomal dominant catecholaminergic polymorphic ventricular tachycardia. Heart Rhythm. 13(8), 1652-1660. https://doi.org/10.1016/j.hrthm.2016.05.004. (2016) (Epub 2016/05/10).

28. Lahat, H. et al. Autosomal recessive catecholamine- or exercise-induced polymorphic ventricular tachycardia: Clinical features and assignment of the disease gene to chromosome 1p13-21. Circulation 103(23), 2822-2827 (2001) (Epub 2001/06/13).

29. Song, L. et al. Calsequestrin 2 (CASQ2) mutations increase expression of calreticulin and ryanodine receptors, causing catecholaminergic polymorphic ventricular tachycardia. J. Clin. Investig. 117(7), 1814-1823. https://doi.org/10.1172/JCI31080. (2007) (Epub 2007/07/04).

30. Rizzi, N. et al. Unexpected structural and functional consequences of the R33Q homozygous mutation in cardiac calsequestrin: A complex arrhythmogenic cascade in a knock in mouse model. Circ. Res. 103(3), 298-306. https://doi.org/10.1161/CIRCRESAHA .108.171660. (2008) (Epub 2008/06/28).

31. Kalyanasundaram, A., Bal, N. C., Franzini-Armstrong, C., Knollmann, B. C. \& Periasamy, M. The calsequestrin mutation CASQ2D307H does not affect protein stability and targeting to the junctional sarcoplasmic reticulum but compromises its dynamic regulation of calcium buffering. J. Biol. Chem. 285(5), 3076-3083. https://doi.org/10.1074/jbc.M109.053892. (2010) (Epub 2009/11/19).

32. Novak, A. et al. Cardiomyocytes generated from CPVTD307H patients are arrhythmogenic in response to beta-adrenergic stimulation. J. Cell Mol. Med. 16(3), 468-482. https://doi.org/10.1111/j.1582-4934.2011.01476.x. (2012) (Epub 2011/11/05).

33. Dirksen, W. P. et al. A mutation in calsequestrin, CASQ2D307H, impairs Sarcoplasmic reticulum $\mathrm{Ca}^{2+}$ handling and causes complex ventricular arrhythmias in mice. Cardiovasc. Res. 75(1), 69-78. https://doi.org/10.1016/j.cardiores.2007.03.002. (2007) (Epub 2007/04/24).

34. di Barletta, M. R. et al. Clinical phenotype and functional characterization of CASQ2 mutations associated with catecholaminergic polymorphic ventricular tachycardia. Circulation 114(10), 1012-1019. https://doi.org/10.1161/CIRCULATIONAHA.106.623793. (2006) (Epub 2006/08/16)

35. Chopra, N. \& Knollmann, B. C. Cardiac calsequestrin: The new kid on the block in arrhythmias. J. Cardiovasc. Electrophysiol. 20(10), 1179-1185. https://doi.org/10.1111/j.1540-8167.2009.01531.x. (2009) (Epub 2009/07/01).

36. Bal, N. C. et al. The catecholaminergic polymorphic ventricular tachycardia mutation R33Q disrupts the $\mathrm{N}$-terminal structural motif that regulates reversible calsequestrin polymerization. J. Biol. Chem. 285(22), 17188-17196. https://doi.org/10.1074/jbc. M109.096354. (2010) (Epub 2010/04/01).

37. Cacheux, M. et al. Interplay between triadin and calsequestrin in the pathogenesis of CPVT in the mouse. Mol. Ther. https://doi. org/10.1016/j.ymthe.2019.09.012. (2019) (Epub 2019/10/15).

38. Josephs, K., Patel, K., Janson, C. M., Montagna, C. \& McDonald, T. V. Compound heterozygous CASQ2 mutations and longterm course of catecholaminergic polymorphic ventricular tachycardia. Mol. Genet. Genomic Med. 5(6), 788-794. https://doi. org/10.1002/mgg3.323. (2017) (Epub 2017/11/28).

39. Landstrom, A. P. et al. Interpreting incidentally identified variants in genes associated with catecholaminergic polymorphic ventricular tachycardia in a large cohort of clinical whole-exome genetic test referrals. Circ. Arrhythm. Electrophysiol. 10(4), e004742. https://doi.org/10.1161/CIRCEP.116.004742. (2017) (Epub 2017/04/14). 
40. Rajagopalan, A. \& Pollanen, M. S. Sudden death during struggle in the setting of heterozygosity for a mutation in calsequesterin 2. Forensic Sci. Med. Pathol. 12(1), 86-89. https://doi.org/10.1007/s12024-015-9733-1. (2016) (Epub 2015/12/17).

41. Liu, B. et al. Ablation of HRC alleviates cardiac arrhythmia and improves abnormal Ca handling in CASQ2 knockout mice prone to CPVT. Cardiovasc. Res. 108(2), 299-311. https://doi.org/10.1093/cvr/cvv222. (2015) (Epub 2015/09/28).

42. Neubauer, J. et al. Post-mortem whole-exome analysis in a large sudden infant death syndrome cohort with a focus on cardiovascular and metabolic genetic diseases. Eur. J. Hum. Genet. 25(4), 404-409. https://doi.org/10.1038/ejhg.2016.199. (2017) (Epub 2017/01/12).

43. Wong, C. H., Koo, S. H., She, G. Q., Chui, P. \& Lee, E. J. Genetic variability of RyR2 and CASQ2 genes in an Asian population. Forensic Sci Int. 192(1-3), 53-55. https://doi.org/10.1016/j.forsciint.2009.07.019. (2009) (Epub 2009/08/28).

44. Liu, N., Ruan, Y. \& Priori, S. G. Catecholaminergic polymorphic ventricular tachycardia. Prog. Cardiovasc. Dis. 51(1), 23-30. https ://doi.org/10.1016/j.pcad.2007.10.005. (2008) (Epub 2008/07/19).

45. Laitinen, P. J., Swan, H. \& Kontula, K. Molecular genetics of exercise-induced polymorphic ventricular tachycardia: Identification of three novel cardiac ryanodine receptor mutations and two common calsequestrin 2 amino-acid polymorphisms. Eur. J. Hum. Genet. 11(11), 888-891. https://doi.org/10.1038/sj.ejhg.5201061 (2003) (Epub 2003/10/23).

46. Wleklinski, M., Parikh, H. \& Knollmann, B. C. An autosomal dominant mutation in calsequestrin 2 causes CPVT withotu changing protein levels. Biophys. J. 116, 95A-A96 (2019).

47. Damiani, E., Volpe, P. \& Margreth, A. Coexpression of two isoforms of calsequestrin in rabbit slow-twitch muscle. J. Mus. Res. Cell Motil. 11, 522-530 (1990).

48. Fliegel, L., Leberer, E., Green, N. M. \& MacLennan, D. H. The fast-twitch muscle calsequestrin isoform predominates in rabbit slow-twitch soleus muscle. FEBS Lett. 242(2), 297-300. https://doi.org/10.1016/0014-5793(89)80488-0. (1989) (Epub 1989/01/02).

49. Biral, D., Volpe, P., Damiani, E. \& Margreth, A. Coexistence of two calsequestrin isoforms in rabbit slow-twitch skeletal muscle fibers. FEBS Lett. 299(2), 175-178. https://doi.org/10.1016/0014-5793(92)80241-8. (1992) (Epub 1992/03/09).

50. Eldar, M., Pras, E. \& Lahat, H. A missense mutation in the CASQ2 gene is associated with autosomal-recessive catecholamine-induced polymorphic ventricular tachycardia. Trends Cardiovasc. Med. 13(4), 148-151. https://doi.org/10.1016/s1050 -1738(03)00025-2. (2003) (Epub 2003/05/07).

51. Viatchenko-Karpinski, S. et al. Abnormal calcium signaling and sudden cardiac death associated with mutation of calsequestrin. Circ. Res. 94(4), 471-477. https://doi.org/10.1161/01.RES.0000115944.10681.EB. (2004) (Epub 2004/01/13).

52. Slupsky, J. R., Ohnishi, M., Carpenter, M. R. \& Reithmeier, R. A. Characterization of cardiac calsequestrin. Biochemistry 26(20), 6539-6544. https://doi.org/10.1021/bi00394a038. (1987) (Epub 1987/10/06).

53. Valle, G. et al. Catecholaminergic polymorphic ventricular tachycardia-related mutations R33Q and L167H alter calcium sensitivity of human cardiac calsequestrin. Biochem. J. 413(2), 291-303. https://doi.org/10.1042/BJ20080163. (2008) (Epub 2008/04/11).

54. Manno, C. et al. Calsequestrin depolymerizes when calcium is depleted in the sarcoplasmic reticulum of working muscle. Proc. Natl. Acad. Sci. USA. 114(4), E638-E647. https://doi.org/10.1073/pnas.1620265114. (2017) (Epub 2017/01/11).

55. Wang, Q. et al. Two pools of IRE1alpha in cardiac and skeletal muscle cells. FASEB J. 33(8), 8892-8904. https://doi.org/10.1096/ fj.201802626R. (2019) (Epub 2019/05/06).

56. Paniagua, R., Royuela, M., Garcia-Anchuelo, R. M. \& Fraile, B. Ultrastructure of invertebrate muscle cell types. Histol. Histopathol. 11(1), 181-201 (1996) (Epub 1996/01/01).

57. Smith, D. S., Gupta, B. L. \& Smith, U. The organization and myofilament array of insect visceral muscles. J. Cell Sci. 1(1), 49-57 (1966) (Epub 1966/03/01)

58. Mackrill, J. J. \& Shiels, H. A. Evolution of excitation-contraction coupling. Adv. Exp. Med. Biol. 1131, 281-320. https://doi. org/10.1007/978-3-030-12457-1_12. (2020) (Epub 2019/10/28).

59. Bal, N. C. et al. The C-terminal calcium-sensitive disordered motifs regulate isoform-specific polymerization characteristics of calsequestrin. Biopolymers 103(1), 15-22. https://doi.org/10.1002/bip.22534. (2015) (Epub 2014/08/06).

60. Miller, M. P. \& Kumar, S. Understanding human disease mutations through the use of interspecific genetic variation. Hum. Mol. Genet. 10(21), 2319-2328. https://doi.org/10.1093/hmg/10.21.2319. (2001) (Epub 2001/11/02).

61. Guo, H. H., Choe, J. \& Loeb, L. A. Protein tolerance to random amino acid change. Proc. Natl. Acad. Sci. USA. 101(25), 9205-9210. https://doi.org/10.1073/pnas.0403255101. (2004) (Epub 2004/06/16).

62. Kumar, S. \& Patel, R. Neutral theory, disease mutations, and personal exomes. Mol. Biol. Evol. 35(6), 1297-1303. https://doi. org/10.1093/molbev/msy085. (2018) (Epub 2018/04/25).

63. Vitkup, D., Sander, C. \& Church, G. M. The amino-acid mutational spectrum of human genetic disease. Genome Biol. 4(11), R72. https://doi.org/10.1186/gb-2003-4-11-r72. (2003) (Epub 2003/11/13).

64. Qin, J. et al. Luminal $\mathrm{Ca}^{2+}$ regulation of single cardiac ryanodine receptors: Insights provided by calsequestrin and its mutants. J. Gen. Physiol. 131(4), 325-334. https://doi.org/10.1085/jgp.200709907. (2008) (Epub 2008/03/19).

65. Houle, T. D., Ram, M. L. \& Cala, S. E. Calsequestrin mutant D307H exhibits depressed binding to its protein targets and a depressed response to calcium. Cardiovasc. Res. 64(2), 227-233. https://doi.org/10.1016/j.cardiores.2004.09.009. (2004) (Epub 2004/10/16).

66. Katz, G. et al. The role of mutant protein level in autosomal recessive catecholamine dependent polymorphic ventricular tachycardia (CPVT2). Biochem. Pharmacol. 86(11), 1576-1583. https://doi.org/10.1016/j.bcp.2013.09.012. (2013) (Epub 2013/09/28).

67. Titus, E. W. et al. The crystal structure of a cardiac calsequestrin filament reveals an atomic mechanism of calsequestrin-associated catecholaminergic polymorphic ventricular tachycardia. bioRxiv. https://doi.org/10.1101/672303 (2019).

68. Louis-Jeune, C., Andrade-Navarro, M. A. \& Perez-Iratxeta, C. Prediction of protein secondary structure from circular dichroism using theoretically derived spectra. Proteins. 80(2), 374-381. https://doi.org/10.1002/prot.23188. (2012) (Epub 2011/11/19).

69. Schoenmakers, T. J., Visser, G. J., Flik, G., \& Theuvenet, A. P. CHELATOR: An improved method for computing metal ion concentrations in physiological solutions. Biotechniques. 12(6):870-4, 6-9. (1992) (Epub 1992/06/01).

70. Campbell, K. P., MacLennan, D. H. \& Jorgensen, A. O. Staining of the $\mathrm{Ca}^{2+}$-binding proteins, calsequestrin, calmodulin, troponin C, and S-100, with the cationic carbocyanine dye "Stains-all". J. Biol. Chem. 258, 11267-11273 (1983).

71. Ashkenazy, H. et al. ConSurf 2016: An improved methodology to estimate and visualize evolutionary conservation in macromolecules. Nucleic Acids Res. 44(W1), W344-W350. https://doi.org/10.1093/nar/gkw408. (2016) (Epub 2016/05/12).

\section{Acknowledgements}

We thank Drs. L. Chen and Z. Tian (Agricultural Life and Environment Sciences, University of Alberta) for help with CD analysis. NLD-His6-KDEL expression vector was a generous gift from Dr. R. Kaufman. This work was supported by the Canadian Institutes of Health Research grants MOP-15291, MOP-15415 and PS-153325 to M.M; MOP-15291, MOP-86750 and PS-153325 to L.B.A.; Natural Sciences and Engineering Research Council of Canada grants to J.B.D., M.M. and L.B.A., SynAD Program and a generous donation from the Kenneth and Sheelagh McCourt family. T.P. is supported by a Women and Children's Health Research Institute studentship and by a studentship award from the Multiple Sclerosis Society of Canada. 


\section{Author contributions}

Q.W., T.P., W.Q. designed experiments, analyzed data and performed biochemical and biophysical experiments. Q.W. also wrote the manuscript. H.J.K. carried out in silico molecular graphic analysis. A.F. and J.B.D. designed experiments and carried out phylogenetic analyses. J.B.D. also wrote the manuscript. X.-Z. C., J.T., L.B.A. and M.M. designed experiments, analyzed data and wrote the manuscript.

\section{Competing interests}

The authors declare no competing interests.

Additional information

Supplementary information is available for this paper at https://doi.org/10.1038/s41598-020-75097-3.

Correspondence and requests for materials should be addressed to J.B.D., L.B.A. or M.M.

Reprints and permissions information is available at www.nature.com/reprints.

Publisher's note Springer Nature remains neutral with regard to jurisdictional claims in published maps and institutional affiliations.

Open Access This article is licensed under a Creative Commons Attribution 4.0 International License, which permits use, sharing, adaptation, distribution and reproduction in any medium or format, as long as you give appropriate credit to the original author(s) and the source, provide a link to the Creative Commons licence, and indicate if changes were made. The images or other third party material in this article are included in the article's Creative Commons licence, unless indicated otherwise in a credit line to the material. If material is not included in the article's Creative Commons licence and your intended use is not permitted by statutory regulation or exceeds the permitted use, you will need to obtain permission directly from the copyright holder. To view a copy of this licence, visit http://creativecommons.org/licenses/by/4.0/.

(C) The Author(s) 2020 